\title{
Median fin function during the escape response of bluegill sunfish (Lepomis macrochirus). I: Fin-ray orientation and movement
}

\section{Citation}

Chadwell, B. A., E. M. Standen, G. V. Lauder, and M. A. Ashley-Ross. 2012. Median Fin Function During the Escape Response of Bluegill Sunfish (Lepomis Macrochirus). I: Fin-Ray Orientation and Movement. Journal of Experimental Biology 215, no. 16: 2869-2880. doi:10.1242/jeb.068585.

\section{Published Version}

doi:10.1242/jeb.068585

\section{Permanent link}

http://nrs.harvard.edu/urn-3:HUL.InstRepos:30510348

\section{Terms of Use}

This article was downloaded from Harvard University's DASH repository, and is made available under the terms and conditions applicable to Other Posted Material, as set forth at http:// nrs.harvard.edu/urn-3:HUL.InstRepos:dash.current.terms-of-use\#LAA

\section{Share Your Story}

The Harvard community has made this article openly available.

Please share how this access benefits you. Submit a story.

\section{Accessibility}




\title{
Median fin function during the escape response of bluegill sunfish (Lepomis macrochirus). I: Fin-ray orientation and movement
}

\author{
Brad A. Chadwell ${ }^{1}$, Emily M. Standen ${ }^{2, *}$, George V. Lauder ${ }^{2}$ and Miriam A. Ashley-Ross ${ }^{1, \dagger}$ \\ ${ }^{1}$ Department of Biology, Box 7325, Wake Forest University, Winston-Salem, NC 27109, USA and ${ }^{2}$ Museum of Comparative Zoology, \\ Harvard University, 26 Oxford Street, Cambridge, MA 02138, USA \\ *Present address: Redpath Museum, McGill University, 859 Sherbrooke Street West, Montreal, Quebec, H3A 0C4, Canada \\ ${ }^{\dagger}$ Author for correspondence (rossma@wfu.edu)
}

\begin{abstract}
SUMMARY
The fast-start escape response is critically important to avoid predation, and axial movements driving it have been studied intensively. Large median dorsal and anal fins located near the tail have been hypothesized to increase acceleration away from the threat, yet the contribution of flexible median fins remains undescribed. To investigate the role of median fins, $\mathrm{C}$-start escape responses of bluegill sunfish (Lepomis macrochirus) were recorded by three high-speed, high-resolution cameras at 500 frames s $^{-1}$ and the 3-D kinematics of individual dorsal and anal fin rays were analyzed. Movement and orientation of the fin rays relative to the body axis were calculated throughout the duration of the C-start. We found that: (1) timing and magnitude of angular displacement varied among fin rays based on position within the fin and (2) kinematic patterns support the prediction that fin rays are actively resisting hydrodynamic forces and transmitting momentum into the water. We suggest that regions within the fins have different roles. Anterior regions of the fins are rapidly elevated to increase the volume of water that the fish may interact with and transmit force into, thus generating greater total momentum. The movement pattern of all the fin rays creates traveling waves that move posteriorly along the length of the fin, moving water as they do so. Flexible posterior regions ultimately act to accelerate this water towards the tail, potentially interacting with vortices generated by the caudal fin during the C-start. Despite their simple appearance, median fins are highly complex and versatile control surfaces that modulate locomotor performance.
\end{abstract}

Supplementary material available online at http://jeb.biologists.org/cgi/content/full/215/16/2869/DC1

Key words: median fin, 3-D kinematics, fin ray, C-start, escape response.

Received 22 November 2011; Accepted 22 April 2012

\section{INTRODUCTION}

Among aquatic predator-prey interactions, the fast-start escape response can determine who eats and who is eaten. Fast-starts are swimming maneuvers associated with the production of high acceleration over a brief period of activity. Most commonly, faststarts are used to evade predators (Eaton et al., 1977; Webb, 1976; Webb, 1978; Weihs, 1973) and capture prey (Hoogland et al., 1956; Rand and Lauder, 1981; Webb and Skadsen, 1980). However, faststarts have also been observed in behaviors such as post-feeding turns (Canfield and Rose, 1993) and social interactions (Fernald, 1975).

Within the kinematic literature, fast-starts of fish have been classified as either C-starts or S-starts based on the shape the body forms at the end of the initial phase of the behavior (reviewed by Domenici and Blake, 1997; Wakeling, 2001). Although S-starts are used by some fish species during escape responses (Harper and Blake, 1990; Schriefer and Hale, 2004; Spierts and Leeuwen, 1999; Webb, 1976), the majority of fish species studied to date have been described as performing the $\mathrm{C}$-start escape response (Domenici and Blake, 1991; Eaton et al., 1977; Webb, 1978). Regardless of type, fast-starts have been divided into three kinematic stages: a preparatory stroke (Stage 1) in which the long axis of the body bends into the characteristic ' $C$ ' or ' $S$ ' shape; a propulsive stroke (Stage 2) that accelerates the fish; and a variable stage (Stage 3) that can include continued swimming strokes, braking maneuvers or simply gliding to a stop (Domenici and Blake, 1997; Weihs, 1973).

The majority of kinematic papers on fast-starts describe the stereotypic maneuver of the axial body and tail, paying particular attention to the timing and magnitude of the turning rate of the head, and the overall performance of the center of mass (displacement, maximum velocity and acceleration). Only a few papers make mention of fin movements, with most simply referring to a qualitative observation that the median fins are splayed (elevated) during the behavior (Eaton et al., 1977; Webb, 1977; Webb, 1978).

Weihs (Weihs, 1973) suggested that enlarged dorsal and anal fins close to the caudal fin could potentially act as a second tail to enhance performance. During the $\mathrm{C}$-start, the axial body rotates to one side (Stage 1), changes direction and then rotates in the opposite direction (Stage 2). During these rotational phases, we would expect the hydrodynamic forces acting on the median fins to correlate with the angular velocity of the body. Therefore, hydrodynamic forces should be greatest during peak angular rotations (Stages 1 and 2) and lowest during the change in direction of axial rotation (Stage 1 to Stage 2 transition) in which angular velocity decreases until it reaches zero. A recent study using particle image velocimetry demonstrated for the first time that the dorsal and anal fins do in fact contribute a thrust component to the acceleration of the fish (Tytell and Lauder, 2008). However, no studies have addressed the movement of fins and how they may be generating this force. 
Prior studies of bluegill have found variation in both the structural design of the dorsal and anal fin rays (Chadwell and Ashley-Ross, 2012) and the mechanical properties of pectoral fin rays (Lauder et al., 2011). Morphological and mechanical differences in structure suggest that different regions of the dorsal and anal fins may play functionally distinct roles, with stiffer and/or less mobile anterior spines and rays primarily supporting the fin membrane and more flexible posterior rays (Chadwell and Ashley-Ross, 2012) undergoing greater undulation. We addressed this question by examining individual fin-ray kinematics during the performance of the escape response in bluegill sunfish, Lepomis macrochirus.

We predict that the timing and magnitude of fin-ray movements should follow those of the body segment to which they are attached. As hydrodynamic forces during the C-start should be greatest at periods of high angular velocity, the phase lag between body and fin movements and degree of fin deflection should be greatest during these periods. As passive resistance to manual lateral deflection has been shown to decrease with position in the median fins (Chadwell and Ashley-Ross, 2012), we predict that the ability of the fin rays to actively resist lateral deflection will also vary with position. Specifically, posterior fin rays will: (1) have increased angular displacement, (2) reach maximum deflection sooner than the anterior fin rays and (3) reverse direction (right-left) later than the anterior rays. Finally, we hypothesize that the soft dorsal and anal fins will show symmetry in their fin-ray kinematics, as the fins demonstrate morphological (Chadwell and Ashley-Ross, 2012) and functional (Lauder et al., 2002; Standen and Lauder, 2005; Tytell, 2006) symmetry during locomotion.

In this study, we show that fin-ray kinematics support the prediction that they are resisting lateral deflection, with the spines and anterior rays demonstrating the greatest ability. Furthermore, fin-ray kinematics vary with position; thus, the surfaces of the dorsal and anal fins do not act as uniform structures. Rather, median fins are highly deformable and complex structures with regional functional roles.

\section{MATERIALS AND METHODS Animals}

Bluegill sunfish, Lepomis macrochirus Rafinesque 1819, collected from seined ponds near Concord, MA, USA, were maintained in individual 40 liter aquaria on a $12 \mathrm{~h}: 12 \mathrm{~h}$ light:dark photoperiod at a mean water temperature of $20^{\circ} \mathrm{C}\left( \pm 1^{\circ} \mathrm{C}\right)$.

\section{Video recording and kinematic analysis}

To reduce the variability in fin kinematics due to variations in the performance of the escape response, it was important that the startle stimulus always occurred from a similar direction. This condition was met when the fish swam at a steady speed, approximately 0.75 total lengths (TL) $\mathrm{s}^{-1}$, in the center of the working volume of a variable speed flow tank $[28 \times 28 \times 80 \mathrm{~cm}$ width $\times$ depth $\times$ length; see fig. 2A in Standen and Lauder (Standen and Lauder, 2005)]. At swimming speeds below $1 \mathrm{TL} \mathrm{s}^{-1}$, bluegill use only their pectoral fins for propulsion and the body, median and caudal fins exhibit minimal movements (Standen and Lauder, 2005). Furthermore, most kinematic and EMG variables do not vary significantly between fast-starts evoked while at a standstill versus when swimming at steady speeds up to $1.6 \mathrm{TL} \mathrm{s}^{-1}$ (Jayne and Lauder, 1993). Additionally, due to the much higher velocities achieved during the C-start $\left(\sim 10 \mathrm{TL} \mathrm{s}^{-1}\right)$ compared with steady swimming $\left(0.75 \mathrm{TL} \mathrm{s}^{-1}\right)$, movement of the fins due to the surrounding flow is presumed to be negligible in comparison to the fin movements generated by the fish.
To be considered for detailed kinematic analysis, a satisfactory C-start required that: (1) the fish held a relatively constant position in the center of the flow tank's working area prior to the presentation of the startle stimulus (size $\mathrm{C}$ battery dropped into the tank behind and to the left of the fish) so that any wall effects were negligible; (2) Stage 2 was completed no later than $60 \mathrm{~ms}$ after onset of the response, ensuring that we were comparing near-maximal performance events; and (3) the entire body was still in view $70 \mathrm{~ms}$ after onset. Detailed analysis was performed on three C-start trials meeting the established criteria recorded from each of three individuals of similar size (mean \pm s.d. $T L=17.1 \pm 1.7 \mathrm{~cm}$, range $=16-19 \mathrm{~cm} ; N=3$ trials each for three fish, nine total trials), out of a total of 46 responses for eight fish. Although the fish performed a right-handed C-start the majority of the time (the initial lateral movement of the head was to the right), two of the nine Cstarts analyzed were left-handed. As all of the numerical parameters measured from the left-handed $\mathrm{C}$-starts fell within the ranges seen in right-handed $\mathrm{C}$-starts, all left-handed $\mathrm{C}$-starts were converted into right-handed $\mathrm{C}$-starts by reversing the sign of all lateral components ( $y$-coordinate) of the sequence to simplify the analysis and discussion of the movements, independent of the handedness of the escape response.

C-starts were recorded with three synchronized high-speed video cameras (two Photron Fastcam $1280 \times 1024$ pixels and a Photron APX system $1024 \times 1024$ pixels; San Diego, CA, USA) at 500 frames s$^{-1}(1 / 2000 \mathrm{~s}$ shutter speed). The cameras were positioned to provide us with clear ventral, dorsal and lateral views of the entire fish throughout the duration of the C-start, enabling us to record the simultaneous movements of the anal and dorsal fins. Fish were allowed to rest a minimum of $30 \mathrm{~min}$ between trials, during which time the circulation of the flow tank was shut off.

Video sequences were analyzed using DigiMat, a custom digitizing program written by Peter Madden for MATLAB (version 7.6.0.324 R2008a, MathWorks, Natick, MA, USA). The three camera images were calibrated and oriented to the shared threedimensional (3-D) volume following a three-step procedure previously described (Standen and Lauder, 2005).

For each sequence, the first frame to show a clear, lateral displacement of the head was noted and the frame prior to this event was designated frame zero $\left(F_{0}\right)$ and time zero $\left(T_{0}\right)$. Pre-startle posture was established by digitizing $F_{0}$ and the preceding nine frames $\left(F_{-9}-F_{-1}\right)$. The succeeding 35 frames $\left(F_{1}-F_{35}\right)$ were digitized to provide a kinematic description throughout the entire duration of Stages 1 and 2 into Stage 3. For each sequence, a total duration of $82 \mathrm{~ms}$ was analyzed $\left(12 \mathrm{~ms}\right.$ prior to $T_{0}$ and $70 \mathrm{~ms}$ post- $\left.T_{0}\right)$. All calculations performed on the 3-D coordinates obtained from Digimat (Madden, 2004) were performed within MATLAB, using custom programs written by B.A.C.

\section{Axial kinematics}

To track axial movements throughout the C-start, 15 points were digitized along the dorsal and ventral midlines, from the tip of the snout to a position above (or below) the caudal peduncle (Fig. 1B). The 15 points were fit to a cubic smoothing spline function to generate the smoothest interpolant to the points with a mean squared error (MSE) of ca. $0.1 \mathrm{~mm}^{3}$ (Walker, 1998), from which 21 evenly spaced points were interpolated. Using these midlines, the body was divided into five segments, each accounting for $20 \%$ of the standard length (SL, distance from snout to caudal peduncle; Fig. 1A). The anterior two segments, the rostrum (Rs) and the operculum (Op), make up the head and the remaining three segments make up the trunk of the fish: the anterior (Ant), middle (Mid) and 

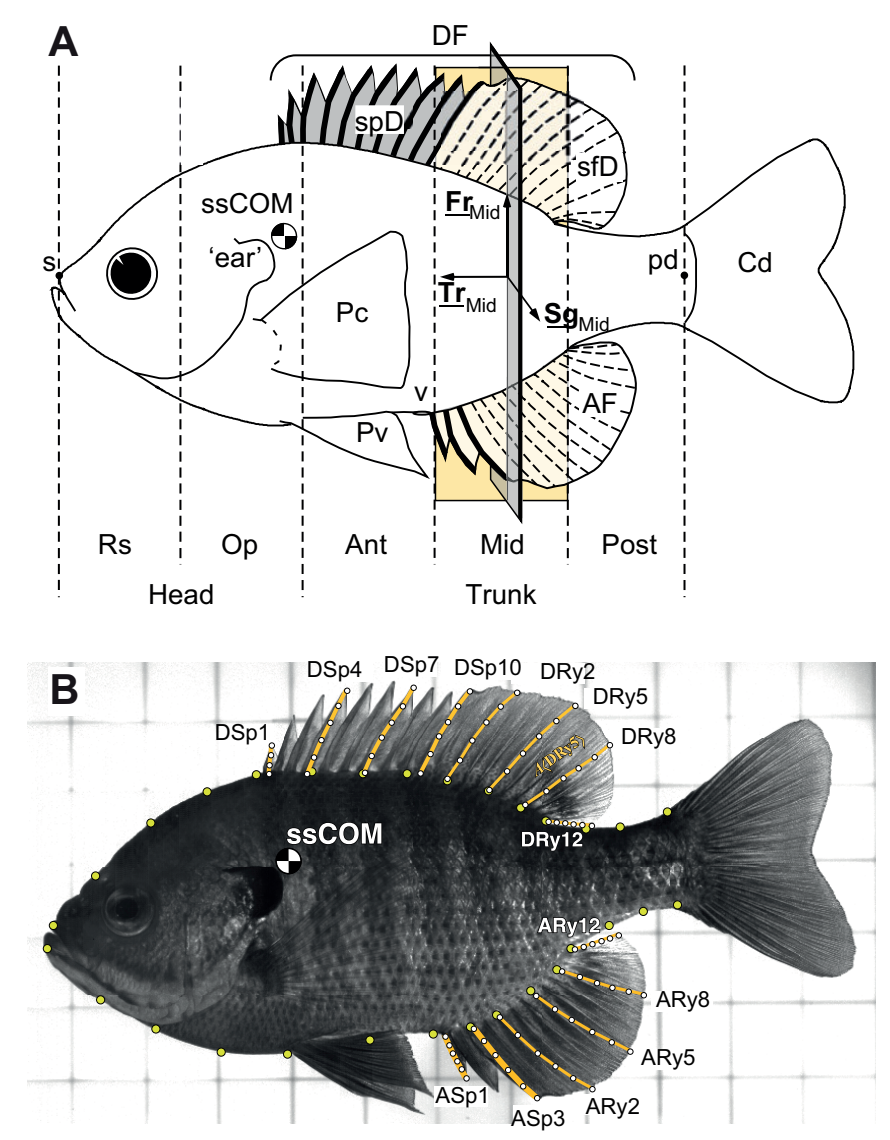

Fig. 1. The bluegill sunfish, Lepomis macrochirus. (A) Illustration of the bluegill. Unpaired median fins: dorsal fins (DF), composed of two developmentally separate fins, the spiny dorsal $(\mathrm{spD})$ and soft dorsal $(\mathrm{sfD})$ fins; anal fin (AF); and caudal fin (Cd). Paired fins: pectoral (Pc) and pelvic $(\mathrm{Pv})$. Fin supports of DF and AF are shown: spines (thick, solid lines) and rays (thin, dashed lines). The body was divided into five equal segments [20\% of the body length from the tip of the snout (s) to the caudal peduncle (pd)]: the rostrum (Rs) and operculum (Op), which comprise the head, and the anterior (Ant), middle (Mid) and posterior (Post) segments of the trunk. Orthogonal axes and planes of the body are shown only for the Mid segment: transverse ( $\underline{\mathbf{r}}_{\text {Mid }}$; grey plane), sagittal ( $\mathbf{S g}_{\text {Mid }}$; orange plane) and frontal ( $\underline{F r}_{\text {Mid }}$; plane not shown). Note that the axes are normal to their corresponding planes. Other symbols: position of the stretched-straight center of mass (ssCOM) indicated by the corresponding symbol, 'ear' of the operculum and vent (v). (B) Still image of the bluegill sunfish. Yellow dots represent the approximate location of the 15 points used to define the dorsal and ventral midlines. Orange lines indicate the digitized fin rays and the approximate location of the four to six points (white dots) used to define each fin ray. The associated text identifies each fin ray based on its location: dorsal (D) versus anal (A), fin-ray type, spine $(\mathrm{Sp})$ versus ray (Ry), and numbered position within the respective fin. $A\langle\mathrm{DRy} 5\rangle$ represents the fin area between rays DRy5 and DRy8.

posterior (Post) segments. This was done in order to compare the orientation and movement of the fins relative to the body segment to which they are attached, e.g. the spiny dorsal fin is primarily attached to Ant and the soft dorsal and anal fins are connected to Mid (Fig. 1B).

For each time point $(t)$ of the sequence, the three orthogonal planes and their corresponding axes (note that the axes are normal to their plane) were defined for each body segment: transverse $\left(\underline{\mathbf{T r}}_{\mathrm{Seg}(\mathrm{t})}\right)$, sagittal $\left(\underline{\mathbf{S g}}_{\operatorname{Seg}(t)}\right)$ and frontal $\left(\underline{\mathbf{F r}}_{\mathrm{Seg}(\mathrm{t})}\right)$, where Seg represents the body segment of interest (Fig. 1A). Relative to the X,Y,Z-coordinate system of the tank, the yaw (rotation about the $\underline{\mathbf{F r}}_{\operatorname{Seg}(\mathrm{t})}$-axis), pitch (rotation about the $\underline{\mathbf{S g}}_{\operatorname{Seg}(t)}$-axis) and roll (rotation about the $\left.\underline{\mathbf{T r}}_{\operatorname{Seg}(t)}\right)^{-}$ axis) angles could be defined and tracked over time for each body segment.

\section{Axial rotation}

Fitting the angle of yaw, $\theta_{\mathrm{t}}$, from each time point to a quintic smoothing spline function (MSE=2.5 deg), the first time derivative of yaw, $\theta_{\mathrm{t}}^{\prime}$, over the duration of the sequence, provided the turning rate (angular velocity, $\mathrm{d} \theta / \mathrm{d} t)$. A positive turning rate $\left(\theta_{\mathrm{t}}^{\prime}>0\right)$ indicates a counterclockwise lateral rotation of the body about $\underline{\mathbf{F r}}_{\mathrm{Seg}(\mathrm{t})}$, with a clockwise rotation resulting in a negative turning rate $\left(\theta_{\mathrm{t}}^{\prime}<0\right)$. A counterclockwise rotation of the head, Rs and Op, are to the left side, but because the axis of rotation of the body lies near the center of mass, a counterclockwise rotation of the trunk segments, Ant, Mid and Post, are to the fish's right side. Thus, when the head and trunk both rotate to the same side of the fish, the sign of $\theta_{\mathrm{t}}^{\prime}$ for the head and trunk segments are reversed. A change in the sign of $\theta_{t}^{\prime}$ indicates a rotational transition of the body segment, i.e. a change in direction.

The turning rate of the rostrum, $\theta_{\mathrm{t}}^{\prime}$, was used to determine the stages of the escape response, according to standard definitions (Domenici and Blake, 1997). Stage $1(\mathrm{~S} 1)$ is the period from $T_{0}$ to the time of the first rotational transition $\left(t \theta_{\mathrm{tr} 1}^{\prime}\right)$, and Stage $2(\mathrm{~S} 2)$ is the period from $t \theta_{\mathrm{tr} 1}^{\prime}$ to the second rotational transition $\left(t \theta_{\mathrm{tr} 2}^{\prime}\right)$. As the rotational transitions of the rostrum define the C-start, $t \theta_{\operatorname{tr} 1}^{\prime}$ and $t \theta_{\mathrm{tr} 2}^{\prime}$ will often be referenced within the text as the $\mathrm{S} 1 / \mathrm{S} 2$ and $\mathrm{S} 2 / \mathrm{S} 3$ transitions, respectively.

To compare the rotation/movement of the fins and their fin rays with the movement of their respective trunk segments, five kinematic variables from $\theta_{\mathrm{t}}^{\prime}$ for Ant and Mid segments were measured: $t \theta_{\max 1}^{\prime}$ and $\theta_{\max 1}^{\prime}$, the time and magnitude, respectively, of the maximum turning rate during the initial rotation of the body segments; $t \theta_{\text {tr }}^{\prime}$, the time of rotational transition; and $t \theta_{\max 2}^{\prime}$ and $\theta_{\max 2}^{\prime}$, the time and magnitude, respectively, of the maximum turning rate during the second rotation of the body segments.

\section{Center of mass}

As this was not a terminal study, the centers of mass for each of the fish used in this study were not measured. Therefore, the location of the centers of mass of several preserved specimens similar in body size to the fish used in this study were measured using the plumb-line method (Vogel, 2003). Among these specimens, the average stretched-straight center of mass (ssCOM) was located just behind and above the 'ear' of the operculum (Fig. 1) at a longitudinal position of ca. $35 \%$ of SL and at a lateral depth of ca. $33 \%$ of the body at that position. For each digitized frame, the position relative to the two midlines that matched the corresponding percent values was used as the 3-D coordinates for the estimated SsCOM of the experimental fish, allowing tracking of a consistent point on the fish's body. The ssCOM coordinates from each time point were fit to a quintic smoothing spline function $\left(\mathrm{MSE}=0.125 \mathrm{~mm}^{3}\right.$ ), allowing for the calculation of displacement, velocity and acceleration of ssCOM over the duration of the C-start. At each time point, the velocity and acceleration of ssCOM was divided into the components parallel and perpendicular to the trajectory of the head at that moment, i.e. $\underline{\operatorname{Tr}}_{R s(t)}$. The components of velocity and acceleration of ssCOM parallel with the heading of the fish are represented by $\mathbf{v}_{\mathrm{t}}$ and $\mathbf{a}_{\mathrm{t}}$, respectively. From the quintic spline, five variables were calculated: displacement of SsCOM at the ends of Stages 1 and 2 $\left(D_{\mathrm{S} 1}\right.$ and $\left.D_{\mathrm{S} 2}\right)$; the maximum velocity achieved during the $\mathrm{C}$-start $\left(\mathbf{v}_{\max }\right)$; and the two peak accelerations $\left(\mathbf{a}_{\max 1}\right.$ and $\left.\mathbf{a}_{\max 2}\right)$. 


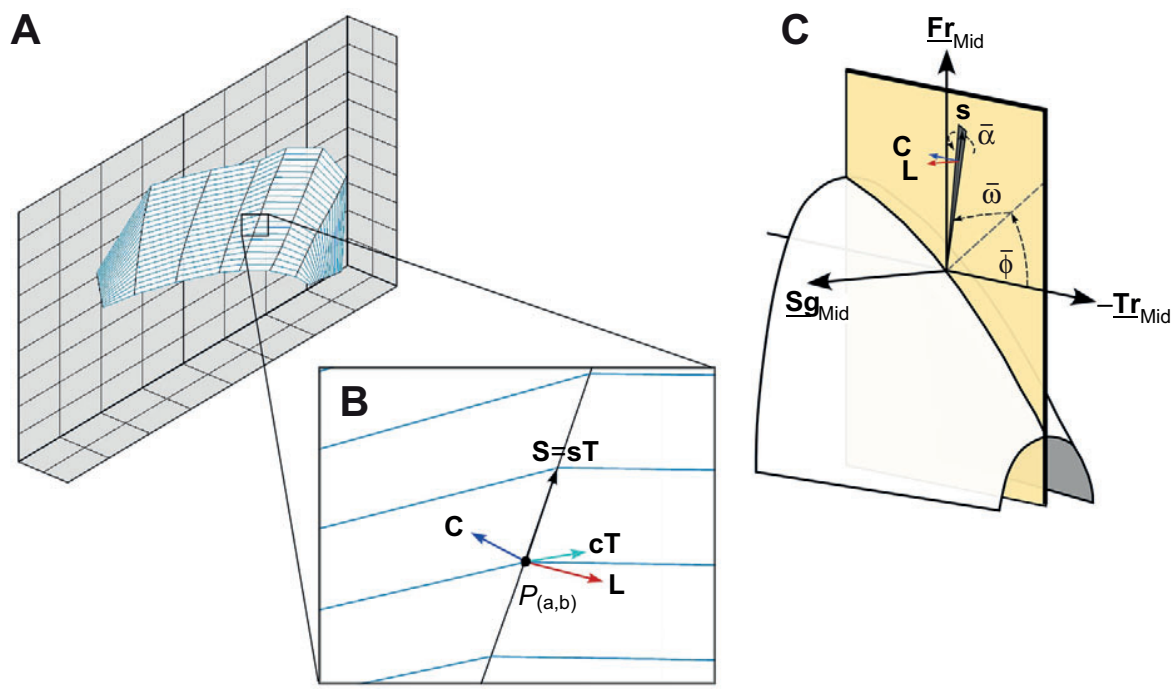

Fig. 2. The reconstructed dorsal fin surface and ray angles. (A) The entire reconstructed dorsal fin. Black lines represent the eight digitized fin rays (the spanwise curves) of the fin. Blue lines represent the chordwise curve of the fin. The intersections of the black and blue lines indicate the 21 interpolated points of each fin ray, from which all measurements were calculated. (B) Close-up of the dorsal fin surface and the vectors calculated at the given point, $P_{(\mathrm{a}, \mathrm{b})}$ : the spanwise (sT) and chordwise (cT) tangents, parallel to their respective curve, and the orthogonal lateral (L), span (S) and chord (C) axes that define the 3-D orientation of the fin surface at $P_{(a, b)}$. (C) Orientation of a dorsal ray relative to the axes of the mid-trunk segment: span axis rotation $(\bar{\alpha})$, sweep $(\bar{\omega})$ and elevation $(\bar{\phi})$. See Fig. 1 for a definition of the body segment axes.
Fin kinematics

Four spines and four rays from the dorsal fins and two spines and four rays from the anal fin were selected for digitizing (Fig. 1B). Throughout the paper, the term 'fin ray' is used to refer to all external skeletal fin supports when the distinction between spines and rays is disregarded. Six points (four in the case of DSp1; Fig. 1B) were digitized along the posterior edge of the selected fin rays, starting proximally at the point at which the fin ray first appears from the skin and musculature and ending at the distal tip. For each fin ray, the six (or four) points were fit to a cubic smoothing spline function $\left(\mathrm{MSE}=0.1 \mathrm{~mm}^{3}\right)$ and 21 equally spaced points along the length of the fin ray curve were interpolated. Thus, point numbers represent the same relative position along the length of each fin ray, e.g. point 1 of all fin rays represents the $0 \%$ position, with each successive point moving distally at $5 \%$ intervals along the length of the fin-ray curve.

\section{Fin surface}

From the coordinates of the 21 interpolated points of every digitized fin ray (both spines and rays), the surface of the entire dorsal and anal fins were reconstructed for each frame (Fig. 2A) and evaluated by fitting all the points to a bivariate tensor function (Kreyszig, 1991), as described in the Appendix of the companion paper (Chadwell et al., 2012).

For each point of the fins, three angles of the fin surface, relative to the reference planes of its associated body segment, were calculated. Span axis rotation, $\alpha$, is the angle of the fin ray about its long axis, relative to the sagittal plane. Sweep, $\omega$, is the angle of the long axis of the fin ray from the sagittal plane, i.e. lateral deflection. Elevation, $\phi$, is the angle between the posterior side of the long axis of the fin ray and the frontal plane (Fig. 2C). A description of these variables and their calculations can be found in the Appendix of the companion paper (Chadwell et al., 2012).

For each fin ray, the average span axis, sweep and elevation angles were calculated from the values of the 21 points along the length of each fin ray to obtain $\bar{\alpha}_{\mathrm{r}(\mathrm{t})}, \bar{\omega}_{\mathrm{r}(\mathrm{t})}$ and $\bar{\phi}_{\mathrm{r}(\mathrm{t})}$. Because of the location of the dorsal and anal fins on opposite midlines, the signs of the angles were adjusted to reflect the direction of fin-ray orientation relative to the fish's body. A positive $\bar{\alpha}_{\mathrm{r}(\mathrm{t})}$ indicates a rotation of the fin ray such that the anterior edge is directed to the left of the sagittal plane. A positive $\bar{\omega}_{\mathrm{r}(\mathrm{t})}$ indicates that the fin ray is directed to the right of the sagittal plane. A positive $\bar{\phi}_{\mathrm{r}(\mathrm{t})}$ represents abduction of the fin ray away from the frontal plane (Fig. 2C).

\section{Fin surface area}

The fin area between adjacent fin rays was calculated by fitting a triangular mesh to the 21 points of the two fin rays and summing the area of each triangle, $A\langle r\rangle_{\mathrm{t}}$, where $\langle r\rangle$ represents the region between fin ray $r$ and the next posterior fin ray, e.g. $A\langle\mathrm{DRy} 5\rangle_{\mathrm{t}}$ indicates the fin area between DRy5 and DRy8 (Fig. 1B). The sum of $A\langle r\rangle_{\mathrm{t}}$ that compose each fin gives the total fin area, $A\langle\text { Fin }\rangle_{\mathrm{t}}$, where Fin represents the specific fin or region: $\mathrm{spD}$, sfD or sfA. Reported areas are true 3-D areas of the fins, not 2-D projected planar values.

\section{Fin-ray kinematics}

For $\bar{\alpha}_{r(t)}$ and $\bar{\omega}_{r(t)}$, five kinematic variables for each sequence were determined: $t \bar{x}_{\max 1}$ and $\bar{x}_{\max 1}$, the time and magnitude, respectively, of maximum $\bar{\alpha}_{r(t)}$ or $\bar{\omega}_{r(t)}$ during the initial rotation of the body segment to which the fin ray is connected; $t \bar{x}_{\text {tr }}$, the time of directional transition, i.e. when $\bar{\alpha}_{r(t)}$ or $\bar{\omega}_{r(t)}$ changes sign or goes to zero; and $t \bar{x}_{\max 2}$ and $\bar{x}_{\max 2}$, the time and magnitude, respectively, of maximum $\bar{\alpha}_{r(t)}$ or $\bar{\omega}_{r(t)}$ during the second rotation of the body segment. From $\bar{\phi}_{\mathrm{r}(\mathrm{t})}, t \bar{\phi}_{\max }$ and $\bar{\phi}_{\max }$, the timing and magnitude of the maximum elevation achieved during the $\mathrm{C}$-start was recorded. From $A\langle r\rangle_{\mathrm{t}}$ and $A\langle\text { Fin }\rangle_{\mathrm{t}}$, only $t A\langle r\rangle_{\max }$ and $t A\langle\text { Fin }\rangle_{\max }$ were reported, the time of maximum area achieved during the $\mathrm{C}$-start.

Based on our null hypothesis, if the fin rays are merely being dragged behind the portion of the trunk to which they are attached, then the timing of their kinematic events should be linked to the timing of the kinematic events of that axial segment, i.e. $t \theta_{\max 1}^{\prime}, t \theta_{\mathrm{tr}}^{\prime}$ and $t \theta_{\max 2}^{\prime}$. As the turning rate of the trunk increases, the hydrodynamic forces acting on the fin surface should also increase, causing the sweep and span axis rotation of the fin rays to be deflected in the opposite direction of trunk rotation, with $t \bar{x}_{\max 1 / 2}$ occurring before $t \theta_{\max 1 / 2}^{\prime}$, and $t \bar{x}_{\text {tr }}$ occurring after $t \theta_{\text {tr }}^{\prime}$. Therefore, the timing of the span axis and sweep events were calculated relative to the timing of the corresponding axial turning rates:

$$
\begin{gathered}
\Delta t \mathrm{x}_{\max 1}=t \mathrm{x}_{\max 1}-t \theta_{\max 1}^{\prime} \\
\Delta t \mathrm{x}_{\mathrm{tr}}=t \mathrm{x}_{\mathrm{tr}}-t \theta_{\mathrm{tr}}^{\prime} \\
\Delta t \overline{\mathrm{x}}_{\max 2}=t \overline{\mathrm{x}}_{\max 2}-t \theta_{\max 2}^{\prime}
\end{gathered}
$$

As there was only a single timing event for fin-ray elevation and fin areas, which did not appear to be associated with maximum turning rate of the body, their timings were adjusted to determine 

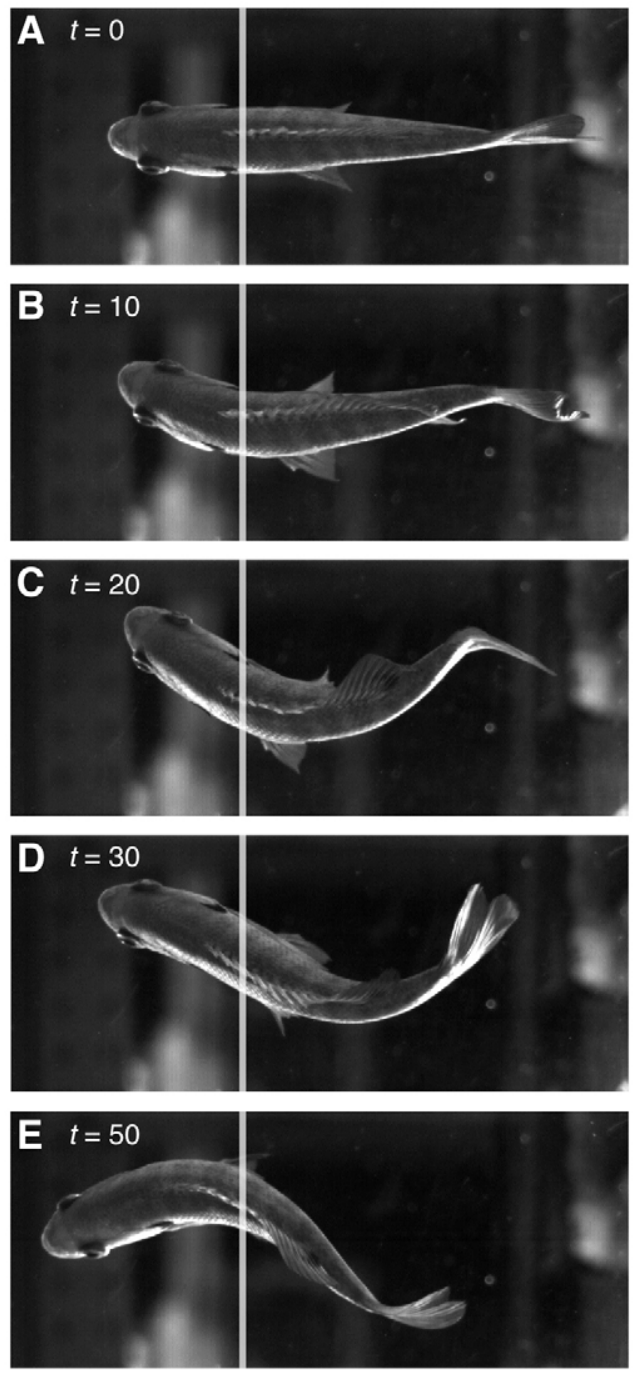

Fig. 3. Stages of a C-start. Still images from the dorsal view of the C-start of a bluegill sunfish at: $(A)$ the frame prior to lateral rotation of the head ( $t=0 \mathrm{~ms}$ ), (B) midway through Stage $1(t=10),(C)$ the end of Stage $1(t=20)$, (D) midway through Stage $2(t=30)$ and $(E)$ the end of Stage $2(t=50)$ Vertical bar represents the initial position of the sSCOM.

whether the time of maximum elevation and area occurred before or after the change in rotational direction of the body segment:

$$
\begin{aligned}
\Delta t \bar{\phi}_{\max } & =t \bar{\phi}_{\max }-t \theta_{\mathrm{tr}}^{\prime}, \\
\Delta t A\langle r\rangle_{\max } & =t A\langle r\rangle_{\max }-t \theta_{\mathrm{tr}}^{\prime}, \\
\Delta t A\langle\text { Fin }\rangle_{\max } & =t A\langle\text { Fin }\rangle_{\max }-t \theta_{\mathrm{tr}}^{\prime} .
\end{aligned}
$$

\section{Statistical analysis}

To test for systematic differences in the C-start performances of the three fish, a one-way multivariate analysis of covariance (MANCOVA) was performed on the 19 axial and SsCOM kinematic variables, using total duration of Stages 1 and $2\left(t \theta_{\mathrm{tr} 2}^{\prime}\right)$ as the covariate.

Excluding ASp1, each digitized fin ray was assigned to one of three groups based on the fin it supported (Fig. 1). The last spine of the dorsal and anal fins (DSp10 and ASp3, respectively) provides the anterior-most support of the soft region of the fins and both are included in the analyses of $\mathrm{SfD}$ and sfA. When referring to the fin rays of the sfD and sfA groups collectively, the two spines will be referred to as $\mathrm{Sp} 0$, due to the discrepancy in their number, and the rays, which are numbered the same, will be referred to as Ry2, Ry5, Ry8 and Ry12.

Although each fin ray is actuated by individual musculature and is capable of independent movement, it is likely that its orientation and movement is influenced by neighboring fin rays because of their connection via the fin membrane. Therefore, ANOVAs were deemed inappropriate to test for differences in kinematics between fin rays within the fins. Instead, the position effect within each fin group was tested using Friedman's method for randomized blocks $\left(\chi^{2}\right)$, using each fish as a block and the fin rays within each group as the treatment levels (Sokal and Rohlf, 1981; Zar, 1984).

To avoid any pseudo-replication from using multiple sequences from each fish, the average value of each kinematic variable was calculated and ranked between fin rays within each group. The ranked average values from each of the three fish were then tested for a position effect in the average timing and magnitude of the finray kinematics. As it is commonly considered that a fast-start escape response requires maximum performance, and the raw values for fin kinematics determine the interaction with the surrounding water, we deemed it important to measure the absolute maxima for each fin-ray parameter. Therefore, we recorded the overall maximum magnitude of each parameter for each fish, and tested the position effect for the ranked maxima of the three fish.

As a means to easily assess the degree of agreement between the three fish for each variable, Kendall's coefficient of concordance $(W)$ was also calculated (Sokal and Rohlf, 1981; Zar, 1984). To determine whether any significant position effects observed within both sfD and sfA were conserved between the two groups, multigroup coefficients of concordance $(\mathcal{W})$ were computed (Zar, 1984).

Wilk's $\lambda$, Friedman's $\chi^{2}$ and Kendall's $W$ and their associated $P$-values were calculated using SPSS v16.0 (IBM, Armonk, NY, USA); a custom program, based on the equations of Zar (Zar, 1984), was written in MATLAB to calculate $\mathscr{W}$ and its $Z$-score. To control for Type I errors resulting from multiple comparisons of the 18 variables of each fin group, $P$-values were compared with corrected $\alpha$-levels using a sequential Bonferroni adjustment (Rice, 1989).

\section{RESULTS}

\section{C-start performance}

A representative escape response sequence is shown in Fig. 3. For the nine $\mathrm{C}$-starts analyzed, kinematic differences among the three fish were consistent and were not statistically significant (Wilk's $\lambda=6.80$, d.f. $=10,2, P=0.135$ ). Mean values for the $20 \mathrm{C}$-start parameters for each of the three fish and the grand means for all sequences are provided in Table 1.

At the end of S1, displacement of ssCOM was minimal, ca. $3 \mathrm{~mm}$, and its velocity was typically only a third of the maximum velocity achieved by the end of S2 (Fig. 4, Table 1). Over the duration of the C-start, two acceleration peaks were consistently observed, the first peak occurring near the $\mathrm{S} 1 / \mathrm{S} 2$ transition, and a second, smaller peak occurring during the latter part of S2 (Fig. 4C).

During S1, both the rostrum and the mid-trunk rotate to the right side of the fish (though their rotational directions are opposite, clockwise versus counterclockwise; Fig. 3B,C) with the second rotational transition of Mid to the left occurring 1-2 ms prior to the end of S1 (Fig. 3D,E, Fig. 5, Table 1). The timing of the maximum turning rates for Mid, $t \theta_{\max 1}^{\prime}$ and $t \theta_{\max 2}^{\prime}$, always occurred during $\mathrm{S} 1$ 
Table 1. Escape response kinematic parameters by fish

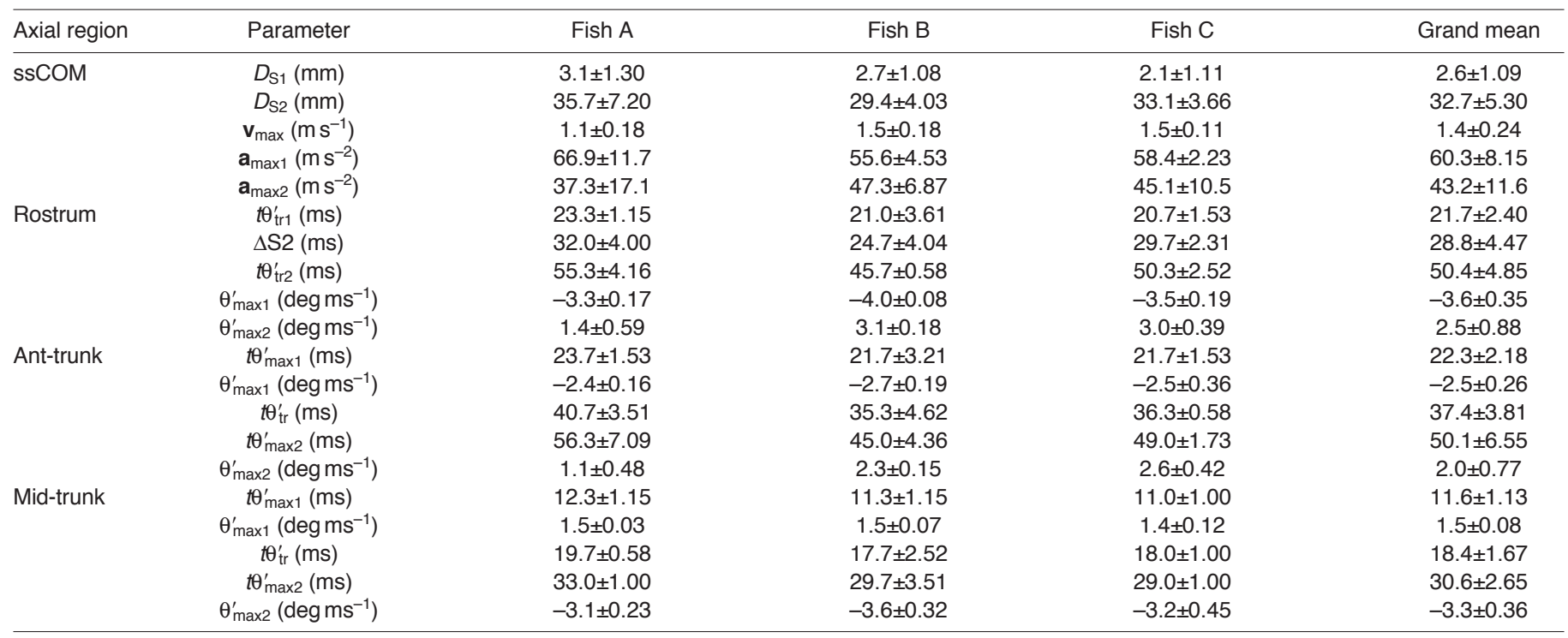

Axial region indicates the region from which the $\mathrm{C}$-start parameters were calculated. See Materials and methods for a description of the parameters. Values are means \pm 1 s.d., $N=3$ sequences for each fish.

and S2, respectively. In the sequence shown, a third rotational transition of Mid, back to the right, occurs several milliseconds prior to the end of S2 (Fig. 5C); however, this was not observed among all sequences (data not shown). Although the timing of the rostrum and mid-trunk were closely coordinated, rotation of the anterior trunk was delayed, with little rotation occurring until midway through $\mathrm{S} 1$, at which point it rotated clockwise to the fish's left with $t \theta_{\text {tr }}^{\prime}$ occurring midway through S2 (Fig. 5B). Timing of the maximum turning rates of Ant, $t \theta_{\max 1}^{\prime}$ and $t \theta_{\max 2}^{\prime}$, was nearly concurrent with the $\mathrm{S} 1 / \mathrm{S} 2$ and S2/S3 transitions (Fig. 5B, Table 1).

\section{Fin-ray kinematics}

The mean and maximum kinematic values for each parameter by fish can be found in the supplementary material. Data for the spines of the spD group can be found in supplementary material Table $\mathrm{S} 1$, and the data for the rays of the soft fins are reported in supplementary material Tables S2 (sfD) and S3 (sfA).

\section{Spiny dorsal fin}

At the onset of the $\mathrm{C}$-start, the spines of the dorsal fin underwent a lateral sweep to the right of the fish with maximum sweep angles occurring $3-15 \mathrm{~ms}$ prior to the first maximum turning rate, $t \theta_{\max 1}^{\prime}$ (Fig. 6A-D, Fig.7A, supplementary material TableS1). Concurrently, a rapid increase in elevation and fin surface area usually preceded any appreciable rotation by the anterior trunk; $\Delta t \bar{\phi}_{\max }, \Delta t A\langle r\rangle_{\max }$ and $\Delta t A\langle s p D\rangle_{\max }$ of the spines all preceded $\mathrm{t} \theta_{\mathrm{tr}}^{\prime}$ with such little difference between spines that they occurred almost simultaneously (Fig. 8A,C, supplementary material TableS1). As $\mathrm{S} 1$ proceeded, a chordwise cupping of the spiny dorsal fin occurred synchronous with a lateral bending of the anterior trunk as the anterior and posterior regions of the fin underwent span axis rotations in opposite directions (anterior spines clockwise, posterior counterclockwise), with concavity for both the fin and body directed to the right (Fig. 3C, Fig. 6F-I, Fig. 7F,G).

By the end of S1 most spines had undergone a directional sweep transition and were now at a sweep angle oriented towards the left of the fish, although Ant was still undergoing its initial rotation to the left (Fig. 7C), which persisted throughout the majority of S2
(Fig. 6A-D). With the exception of DSp10, maximum sweep to the left by the anterior three spines always preceded $t \theta_{\max 2}^{\prime}$ by several milliseconds (Fig.7D). Midway through S2, span axis rotation underwent a direction transition followed by a second chordwise cupping of the fin, matching the lateral bend of the anterior trunk, directed towards the left (Fig. 3E, Fig. 6F-I, Fig. 7I,J). Elevation and fin area during S2 was highly variable between sequences, either maintaining the same degree of elevation/fin area or depressing the spines/decreasing fin area, which may or may not be followed by a second elevation and increase of fin area (data not shown).
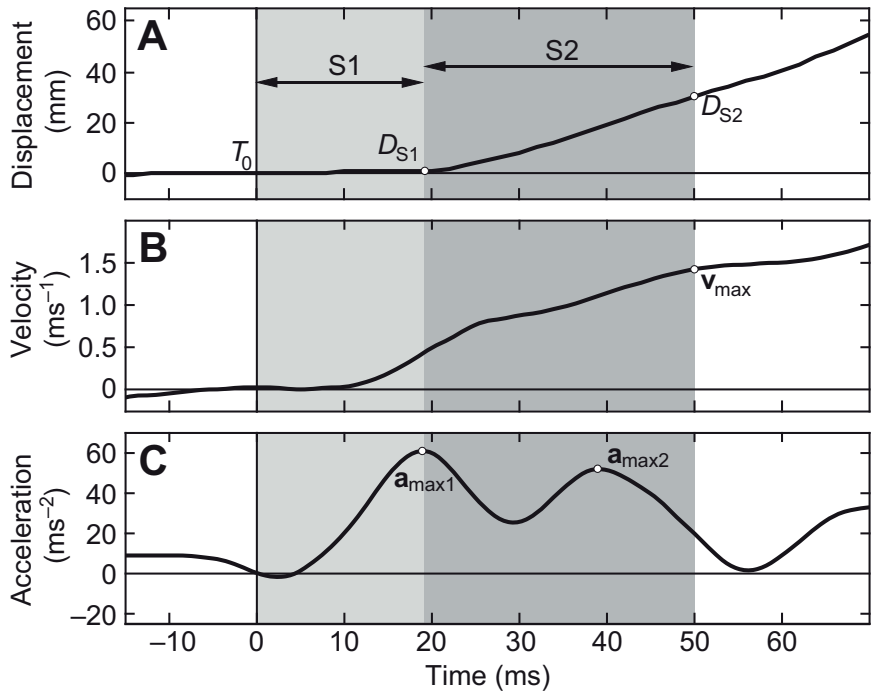

Fig. 4. Center of mass kinematics throughout a C-start. (A) Displacement. $D_{\mathrm{S} 1}$ and $D_{\mathrm{S} 2}$, SsCOM displacement at the end of Stages 1 and 2, respectively. (B) Velocity. $\mathbf{v}_{\max }$, maximum ssCOM velocity achieved during the two stages. (C) Acceleration. $\mathbf{a}_{\max 1}$ and $\mathbf{a}_{\max 2}$, the two peak ssCOM accelerations during the two stages. In all panels, the light gray region indicates the period of Stage 1 (S1), starting from $T_{0}$ (onset of C-start), and the darker gray region indicates the period of Stage 2 (S2). All time sequences shown are from the same fish. 


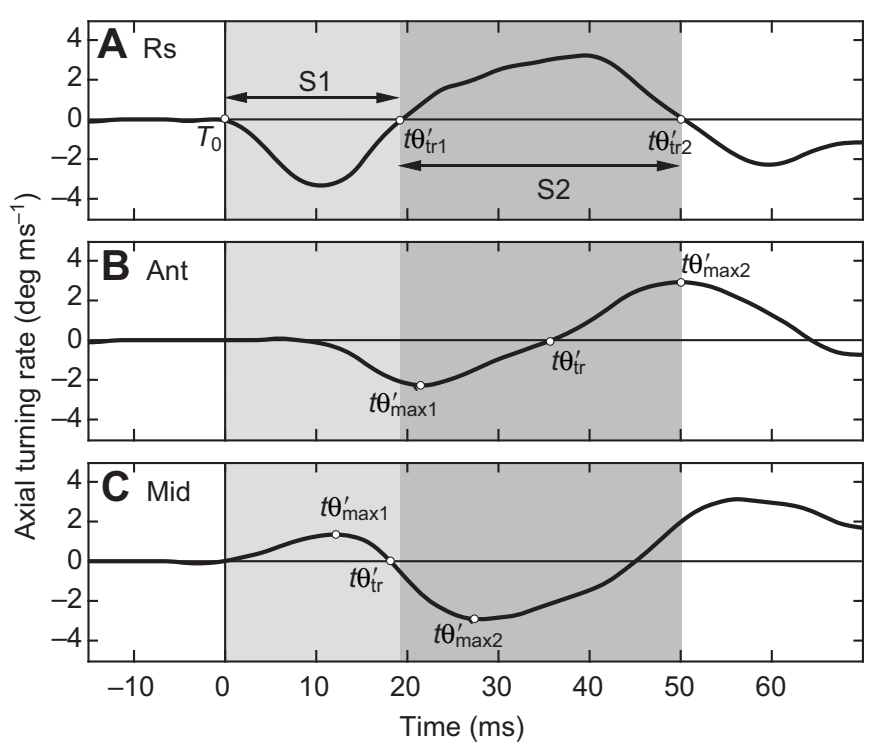

Fig. 5. Axial turning rate throughout a C-start. (A) Turning rate of the rostrum with the two rotational transitions, $t \theta_{\mathrm{tr} 1}^{\prime}$ and $t \theta_{\mathrm{tr} 2}^{\prime}$, indicated (white dots). Stage 1 (S1), time period between $T_{0}$ (time zero) and $t \theta_{\text {tr1 }}^{\prime}$; Stage 2 (S2), time period between $t \theta_{\mathrm{tr} 1}^{\prime}$ and $t \theta_{\mathrm{tr} 2}^{\prime}$. (B) Turning rate of the anterior trunk with the time of the two maximal rotations and rotational transition, $t \theta_{\max 1}^{\prime}, t \theta_{\max 2}^{\prime}$ and $t \theta_{\mathrm{tr}}^{\prime}$, indicated. (C) Turning rate of the middle trunk with its three kinematic events, $t \theta_{\max 1}^{\prime}, t \theta_{\mathrm{tr}}^{\prime}$ and $t \theta_{\max 2}^{\prime}$, indicated. Shaded regions as in Fig. 4.

Although $\bar{\omega}_{\max 1}$ and $\bar{\omega}_{\max 2}$ of the anterior three spines were minimal $(<10 \mathrm{deg})$, maximum sweep angles of DSp10 were typically two to three times greater (Fig. 7B,E). Despite the high degree of concordance among the fish, particularly for the initial sweep parameters $(W>0.90)$, and the trend of increasing $\Delta t \bar{\omega}_{\max 1}$ and $\bar{\omega}_{\max 1}$ with spine position, no significant position effects were found among the sweep parameters due to the $\alpha$-level adjustment $\left(\Delta t \bar{\omega}_{\max 1}, \bar{\omega}_{\max 1}\right.$, $\omega_{\max 1}, \Delta t \bar{\omega}_{\text {tr }}:$ all $W=0.91, \chi^{2}=8.20, P=0.017 ; \Delta t \bar{\omega}_{\max 2}: W=0.73$, $\chi^{2}=6.60, P=0.075 ; \bar{\omega}_{\max 2}, \omega_{\max 2}$ : both $W=0.64, \chi^{2}=5.80, P=0.148$; supplementary material Table $\mathrm{S} 4$ ).

Unlike the timing of sweep angles, average timing of all three span axis events occurred concurrently with the turning rate events of Ant, within ca. $\pm 5 \mathrm{~ms}$ (Fig. 7F,H,I, supplementary material TableS1), with neither a significant position effect $\left(\Delta t \bar{\alpha}_{\max 1}: W=0.78\right.$, $\chi^{2}=7.00, P=0.054 ; \Delta t \bar{\alpha}_{\text {tr }}: W=0.24, \chi^{2}=0.53, P=0.608 ; \Delta t \bar{\alpha}_{\max 2}$ : $W=0.29, \chi^{2}=2.60, P=0.524$; supplementary material TableS4) nor a clear pattern among the spine position. However, a significant position effect was found for $\bar{\alpha}_{\max 1}$ and $\bar{\alpha}_{\max 2}$ for both the mean and maximal fish values $\left(\bar{\alpha}_{\max 1}, \alpha_{\max 1}, \bar{\alpha}_{\max 2}, \alpha_{\max 2}\right.$ : all $W=1.00$, $\chi^{2}=9.00, P=0.002$; supplementary material Table S4).

Timing of maximum spine elevation and fin area showed no position effect $\left(\Delta t \bar{\phi}_{\max }: W=0.66, \chi^{2}=5.90, P=0.115 ; \Delta t A\langle r\rangle_{\max }\right.$ : $W=0.11, \chi^{2}=0.67, P=0.944$; supplementary material Table S4). However, $\bar{\phi}_{\max }$ was significantly related to position, with mean and maximal $\bar{\phi}_{\max }$ decreasing with spine position (for both, $W=1.00$, $\chi^{2}=9.00, P=0.002$; supplementary material Table S4, Fig. 8B). Change in spine elevation and fin area after the initial increase varied among the sequences, with three generalized patterns emerging: maintenance of maximum elevation/fin area, decrease in the elevation/fin area that persisted throughout the rest of the C-start, or a transient depression/decrease followed by an increase in elevation/fin area (data not shown).
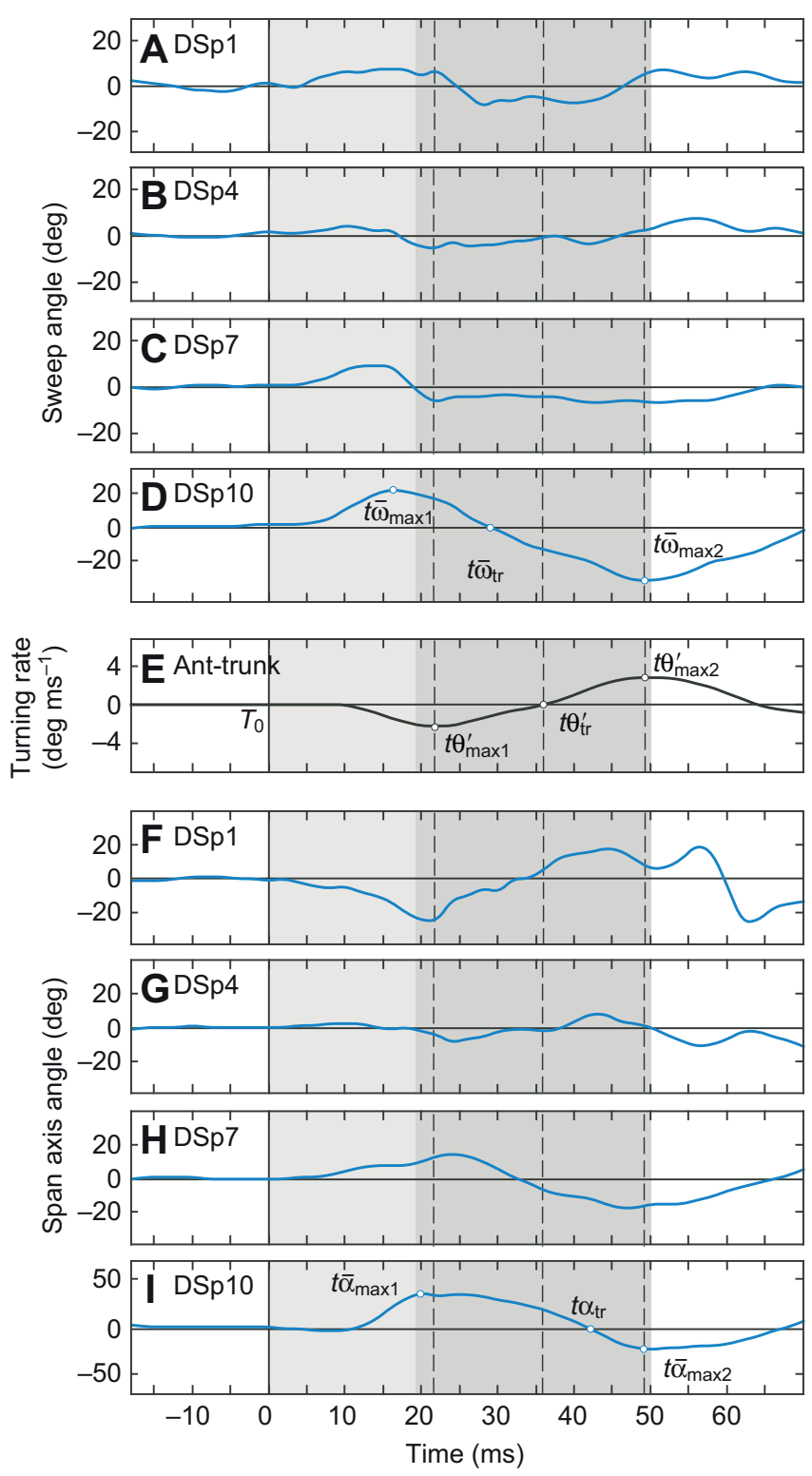

Fig. 6. Sweep and span axis angles of the spiny dorsal fin rays. (A-D) Sweep angles of individual spines. The time of the two maximum sweep angles and directional transition, $t \bar{\omega}_{\max 1}, t \bar{\omega}_{\max 2}$ and $t \bar{\omega}_{\mathrm{tr}}$, are indicated for one spine (DSp10). (E) Turning rate of Ant, shown to provide reference for the movements of the associated fin rays. (F-I) Span axis angles of individual spines. The time of the two maximum span axis angles and directional transition, $t \bar{\alpha}_{\max 1}, t \bar{\alpha}_{\max 2}$ and $t \bar{\alpha}_{t r}$, are indicated for one spine (DSp10). Shaded regions as in Fig. 4.

\section{Soft dorsal and anal fins}

As with the spines, the fin rays were erected and fin area increased soon after the onset of the $\mathrm{C}$-start, usually reaching maximum values by the end of S1 (Fig. 9). As Mid rotated towards the right, the fin rays underwent a sweep to the left, with the time and magnitude of maximum sweep increasing posteriorly, similar to a traveling wave (Figs 9, 10). Likewise, a wave in clockwise span axis rotation occurred along the longitudinal length of the fins (Fig.9). During the initiation of this wave of span axis rotation within the anterior fin region, a brief counterclockwise span axis rotation of Ry12 occurred, which resulted in a transient chordwise cupping of the posterior fin, directed to the right. 


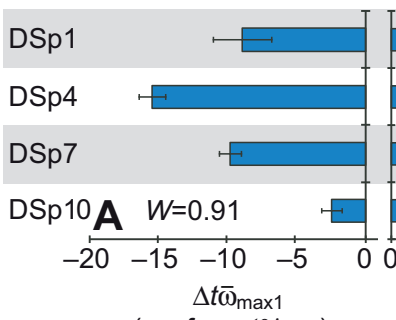

(ms from $t \theta_{\max 1}^{\prime}$ )

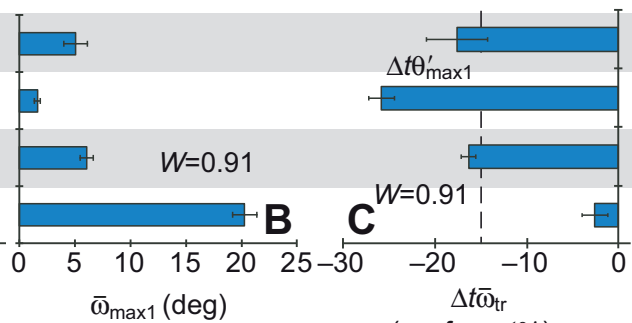

(ms from $\left.t \theta_{\mathrm{tr}}^{\prime}\right)$

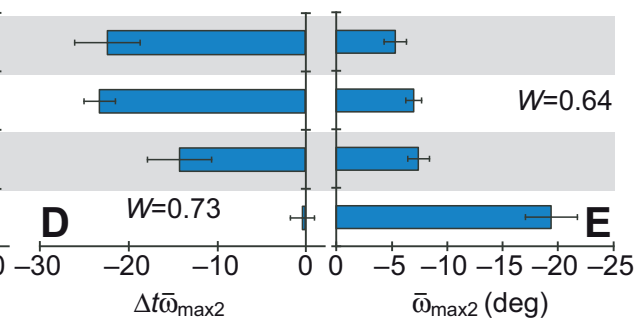

(ms from $\left.t \theta_{\max 2}^{\prime}\right)$

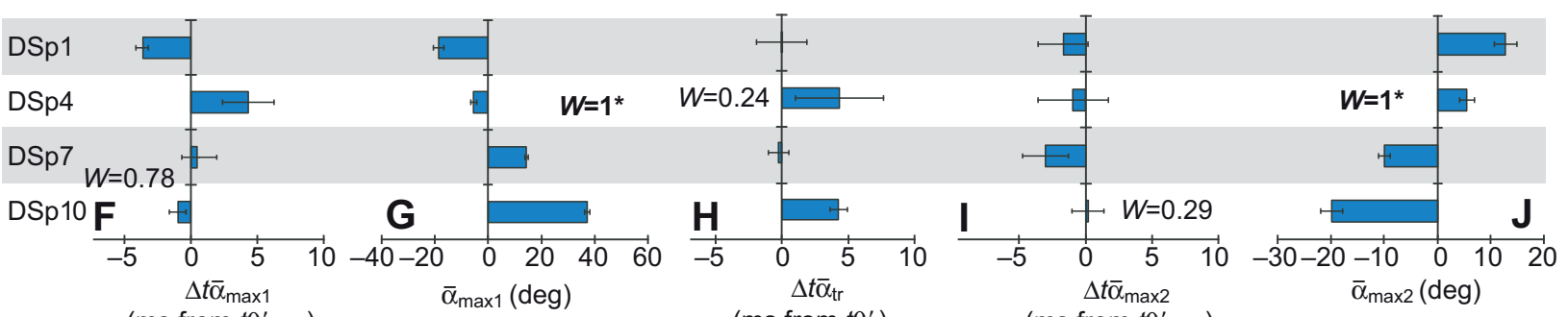

(ms from $\left.t \theta_{\max 1}^{\prime}\right)$

(ms from $\left.t \theta_{\mathrm{tr}}^{\prime}\right)$

(ms from $t \theta_{\max 2}^{\prime}$ )

Fig. 7. Average sweep and span axis parameters of the spiny dorsal fin rays. (A) Timing of the first maximum sweep angle. (B) First maximum sweep angle. (C) Timing of the directional sweep transition. (D) Timing of the second maximum sweep angle. (E) Second maximum sweep angle. (F) Timing of the first maximum span axis angle. (G) First maximum span axis angle. (H) Timing of the directional span axis transition. (I) Timing of the second maximum span axis angle. (J) Second maximum span axis angle. All timing parameters are in relation to the corresponding time event of the Ant-trunk turning rate, $t \theta^{\prime}$ event. The dashed line in $\mathrm{C}$ represents the average time difference between the first maximum turning rate and the rotational transition of the anterior trunk, $\Delta t \theta_{\max 1}^{\prime}=t \theta_{\max 1}^{\prime}-t \theta_{\mathrm{tr}}^{\prime}$. For each parameter, Kendall's coefficient of concordance, $W$, is provided; significant position effects are indicated in bold with an asterisk. Bars are means \pm 1 s.e.m., $N=9$ sequences from three fish.

At the end of S1, Mid had undergone its transition and rotation was now directed to the left. The fin rays underwent a rapid sweep transition, with their sweep now oriented to the right. As with S1, the time and magnitude of maximum sweep increased with position (Figs 9, 10). The span axis rotation of the fin rays also changed direction, resulting in a slight cupping of the posterior soft fin to the left (Fig. 9). As with spD, fin-ray elevation and fin area during S2 varied among sequences.

For both fin groups, the time difference between each sweep $(\omega)$ event (max1, tr and max2) and their corresponding Mid-trunk $t \theta^{\prime}$ events increased with fin-ray position (Fig. 10A,C,D, supplementary material Tables S5-S7). All three time parameters showed a

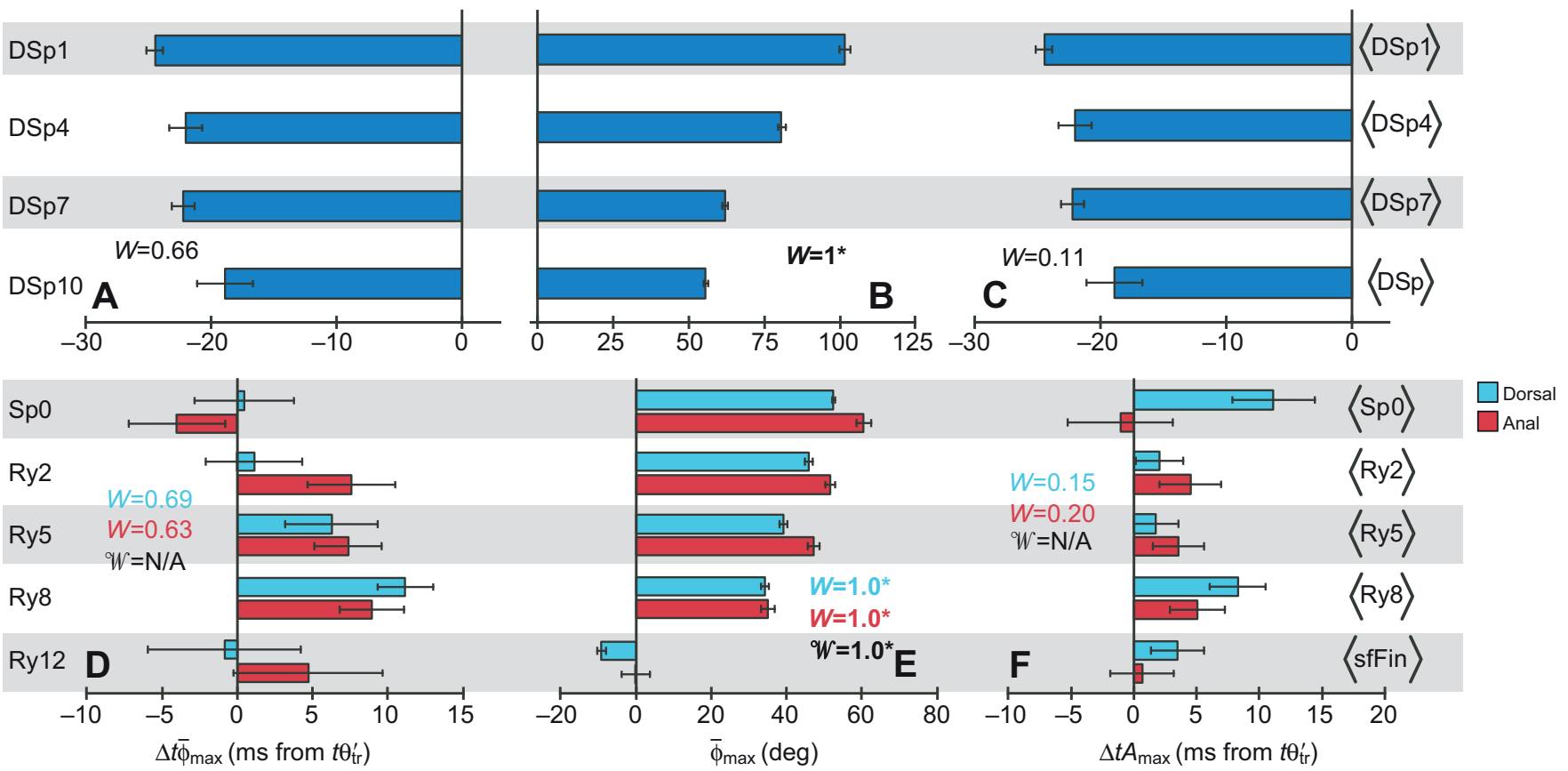

Fig. 8. Average elevation and fin area parameters of the median fin rays. (A) Timing of maximum dorsal spine elevation. (B) Maximum dorsal spine elevation. (C) Timing of maximum dorsal spiny fin areas. (D) Timing of maximum soft fin-ray elevation. (E) Maximum soft fin-ray elevation. (F) Timing of maximum soft fin areas. Symbols as in Fig. 7. 
significant position effect within $\operatorname{sfD}\left(\Delta t \bar{\omega}_{\max 1}: W=0.96, \chi^{2}=11.47\right.$, $P=0.001 ; \Delta t \bar{\omega}_{\mathrm{tr}}: W=1.00, \chi^{2}=12.00, P<0.001 ; \Delta t \bar{\omega}_{\max 2}: W=0.96$, $\chi^{2}=11.47, P=0.001$; supplementary material Table S5); within sfA, a significant position effect was found in $\Delta t \bar{\omega}_{\max 1}$ and $\Delta t \bar{\omega}_{\mathrm{tr}}$, but not $\Delta t \bar{\omega}_{\max 2}\left(\Delta t \bar{\omega}_{\max 1}\right.$ and $\Delta t \bar{\omega}_{\mathrm{tr}}:$ both $W=0.95, \chi^{2}=11.44, P=0.001 ;$
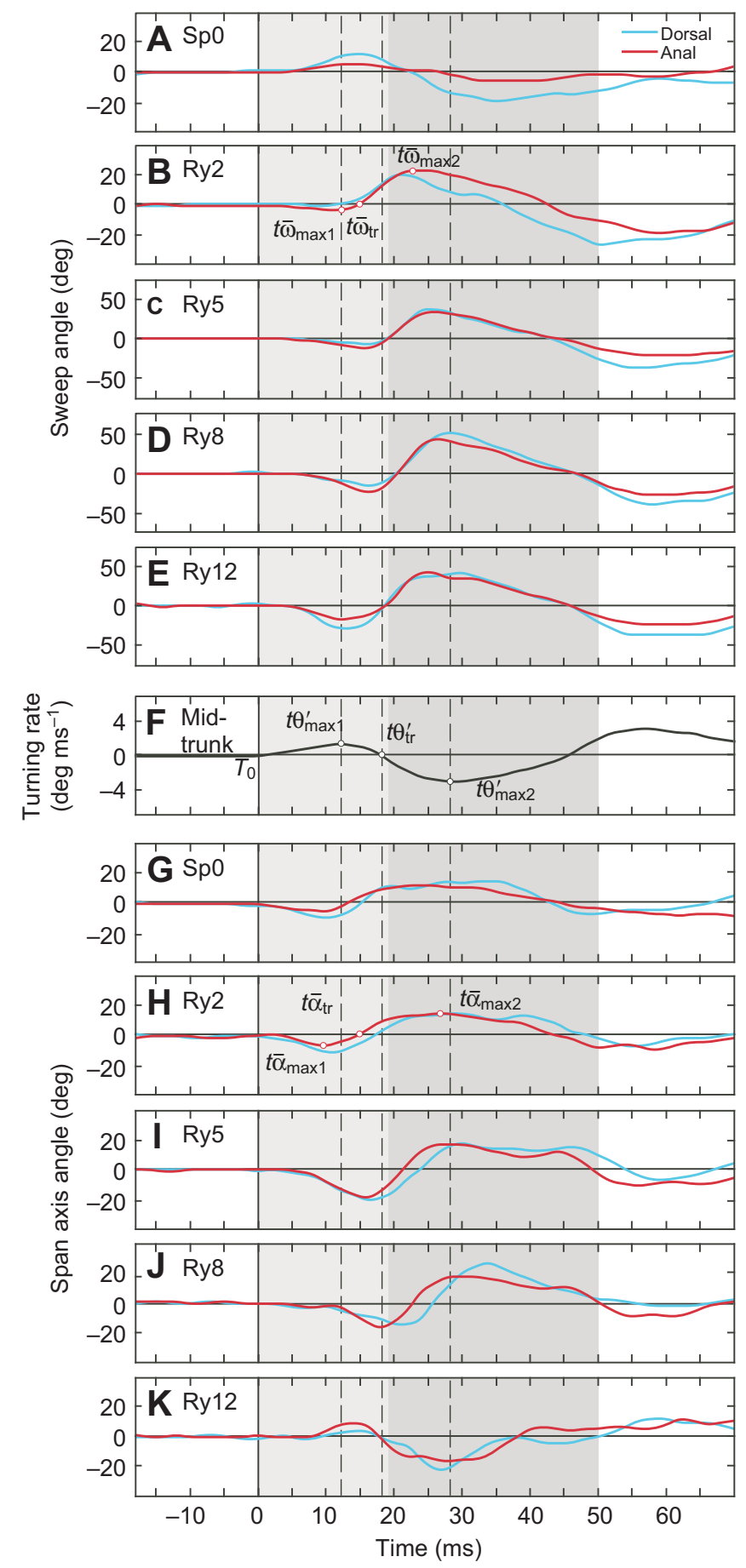

Fig. 9. Sweep and span axis angles of the soft dorsal (blue) and anal (red) fin rays. (A-E) Sweep angles of individual fin rays. The time of the two maximum sweep angles and directional transition, $t \bar{\omega}_{\max 1}, t \bar{\omega}_{\max 2}$ and $t \bar{\omega}_{\mathrm{tr}}$, are indicated for one fin ray (ARy2). (F) Turning rate of Mid, shown to provide reference for the movements of the associated fin rays. (G-K) Span axis angles of individual fin rays. The time of the two maximum span axis angles and directional transition, $t \bar{\alpha}_{\max 1}, t \bar{\alpha}_{\max 2}$ and $t \bar{\alpha}_{\mathrm{tr}}$, are indicated for one ray (ARy2). Shaded regions as in Fig. 4.
$\Delta t \bar{\omega}_{\max 2}: \quad W=0.69, \chi^{2}=8.27, P=0.056 ;$ supplementary material Table S6). Both $\bar{\omega}_{\max 1}$ and $\bar{\omega}_{\max 2}$ increased with position within sfD and sfA (Fig. 10B,E), with significant position effects for both the mean and maximal values ( $\mathrm{sfD} \bar{\omega}_{\max 1}, \omega_{\max 1}, \bar{\omega}_{\max 2}$ : all $W=0.96$, $\chi^{2}=11.47, \quad P=0.001 ; \quad \omega_{\max 2}: \quad W=0.89, \quad \chi^{2}=10.67, \quad P=0.004 ;$ supplementary material Table S5; sfA $\bar{\omega}_{\max 1}, \omega_{\max 1}$ : both $W=0.97$, $\chi^{2}=11.59, P<0.001 ; \bar{\omega}_{\max 2}, \omega_{\max 2}$ : both $W=1.00, \chi^{2}=12.00, P<0.001$; supplementary material Table S6). For all sweep parameters in which significant position effects were found in both the sfD and sfA groups, a significant degree of concordance between groups was also found $\left(\Delta t \bar{\omega}_{\max 1}:{ }^{W}=0.93, Z=5.57, P<0.001 ; \Delta t \bar{\omega}_{\text {tr }}, \bar{\omega}_{\max 2}\right.$ : both $\mathscr{W}=0.97, Z=5.80, P<0.001 ; \bar{\omega}_{\max 1}, \omega_{\max 1}$ : both $\mathscr{W}=0.96, Z=5.73$, $P<0.001 ; \omega_{\max 2}: \mathcal{W}=0.93, Z=5.60, P<0.001$; supplementary material TableS7).

Among the first four fin rays, the span axis parameters $\Delta t \bar{\alpha}_{\max 1}$, $\Delta t \bar{\alpha}_{\text {tr }}$ and $\Delta t \bar{\alpha}_{\max 2}$ increased with position, whereas the timing of Ry12 usually occurred at the same time as Sp0 or Ry2 (Fig. 10F,H,I, supplementary material Tables S2, S3). Significant position effects were found for $\Delta t \bar{\alpha}_{\max 1}$ and $\Delta t \bar{\alpha}_{\mathrm{tr}}$, but not $\Delta t \bar{\alpha}_{\max 2}$ for both $\mathrm{sfD}$ $\left(\Delta t \bar{\alpha}_{\max 1}: W=0.96, \chi^{2}=11.47, P=0.001 ; \Delta t \bar{\alpha}_{\mathrm{tr}}: W=1.00, \chi^{2}=12.00\right.$, $P<0.001 ; \Delta t \bar{\alpha}_{\max 2}: W=0.82, \chi^{2}=9.87, P=0.015$; supplementary material Table S5) and sfA $\left(\Delta t \bar{\alpha}_{\max 1}, \Delta t \bar{\alpha}_{\mathrm{tr}}:\right.$ both $W=0.95, \chi^{2}=11.44$, $P=0.001 ; \Delta t \bar{\alpha}_{\max 2}: W=0.82, \chi^{2}=9.87, P=0.015$; supplementary material Table S6). During the initial span axis rotation, clockwise $\bar{\alpha}_{\max 1}$ increased with fin-ray position among Sp0, Ry2 and Ry5. At Ry8, clockwise $\bar{\alpha}_{\max 1}$ decreased and by Ry12, $\bar{\alpha}_{\max 1}$ was directed counterclockwise. A similar pattern in the reverse direction was observed for $\bar{\alpha}_{\max 2}$ (Fig. 10G,J). For both sfD and sfA, mean $\bar{\alpha}_{\max 1}$, but not maximal, was significantly related to position $\left(\mathrm{sfD} \bar{\alpha}_{\max 1}\right.$ : $W=0.87, \chi^{2}=10.40, P=0.001 ; \alpha_{\max 1}: W=0.73, \chi^{2}=8.80, P=0.038$; supplementary material TableS5; sfA $\bar{\alpha}_{\max 1}: W=0.87, \chi^{2}=10.40$, $P=0.005 ; \alpha_{\max 1}: W=0.78, \chi^{2}=9.33, P=0.026$; supplementary material TableS6). In sfD, but not sfA, significant position effects were found for both mean and maximal $\bar{\alpha}_{\max 2}\left(\mathrm{sfD} \bar{\alpha}_{\max 2}: W=0.89, \chi^{2}=10.67\right.$, $P=0.004 ; \alpha_{\max 2}: W=0.96, \chi^{2}=11.47, P=0.001 ;$ supplementary material TableS5; sfA $\bar{\alpha}_{\max 2}: W=0.60, \chi^{2}=7.20, P=0.117 ; \alpha_{\max 2}$ : $W=0.56, \chi^{2}=6.67, P=0.163$; supplementary material TableS6). For all span axis parameters in which significant position effects were found in both the sfD and sfA fin groups, a significant degree of concordance between sfD and sfA was also found $\left(\Delta t \bar{\alpha}_{\max 1}, \Delta t \bar{\alpha}_{\mathrm{tr}}\right.$ : both $\mathscr{W}=0.97, Z=5.80, P<0.001 ; \bar{\alpha}_{\max 1}: \mathcal{W}=0.87, Z=5.20, P=0.000$; supplementary material Table $\mathrm{S} 7$ ).

Timing of maximal elevation $(\phi)$ and area were highly variable between fin rays of the soft dorsal and anal fins and no significant position effects were found for either group (sfD $\Delta t \bar{\phi}_{\max }: W=0.69$, $\chi^{2}=8.27, \quad P=0.056 ; \quad \Delta t A\langle r\rangle_{\max }: \quad W=0.15, \quad \chi^{2}=1.35, \quad P=0.781$; supplementary material Table S5; sfA $\Delta t \bar{\phi}_{\max }: W=0.63, \chi^{2}=7.52$, $P=0.083 ; \Delta t A\langle r\rangle_{\max }: W=0.20, \chi^{2}=1.80, P=0.727$; supplementary material TableS6). For both groups, $\bar{\phi}_{\max }$ decreased with fin-ray position (Fig. 8E, supplementary material Tables S2, S3) and significant position effects for both mean and maximal $\bar{\phi}_{\max }$ were found in both $\mathrm{sfD}\left(\bar{\phi}_{\max }, \phi_{\max }\right.$ : both $W=1.00, \chi^{2}=12.00, P<0.001$; supplementary material Table S5) and sfA ( $\bar{\phi}_{\max }, \phi_{\max }$ : both $W=1.00$, $\chi^{2}=12.00, P<0.001$; supplementary material Table S6) as well as a significant degree of concordance between sfD and sfA $\left(\bar{\phi}_{\max }, \phi_{\max }\right.$ : both $\mathcal{W}=1.00, Z=6.00, P<0.001$; supplementary material Table S7).

\section{DISCUSSION}

During locomotion, fin-ray kinematics are the result of the interaction between the fin ray's inherent structural properties and its resistance/susceptibility to the combination of forces acting on it: external hydrodynamic forces, activity of its intrinsic muscles, 


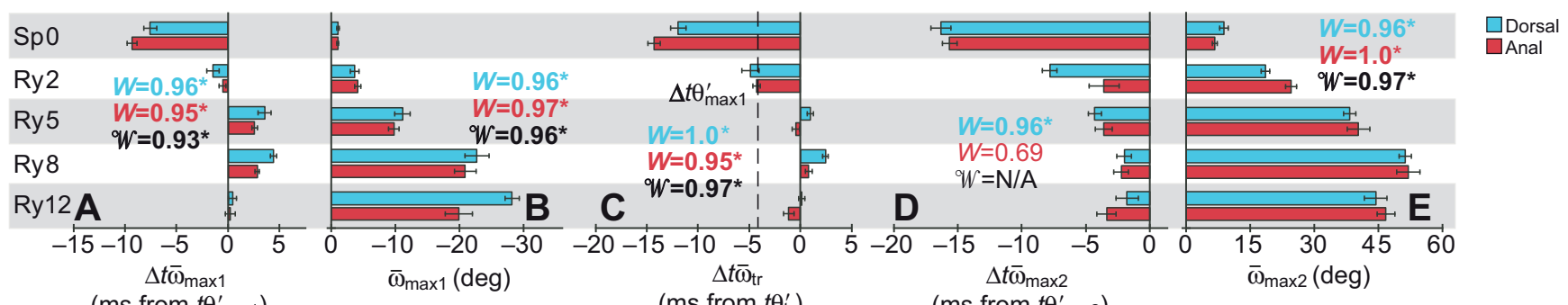

(ms from $\left.t \theta_{\max 1}^{\prime}\right)$

$\left(\mathrm{ms}\right.$ from $\left.t \theta_{\mathrm{tr}}^{\prime}\right)$

(ms from $\left.t \theta_{\max 2}^{\prime}\right)$

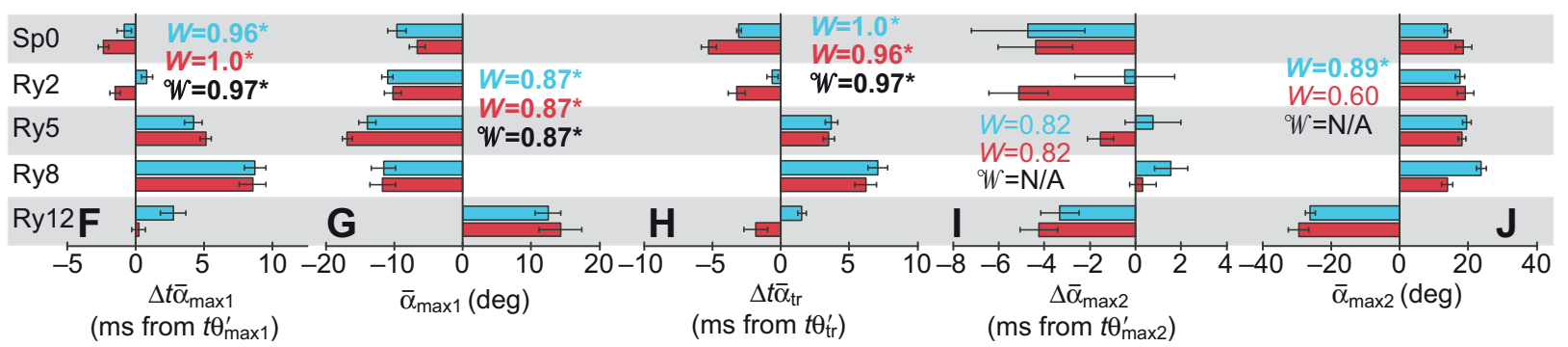

Fig. 10. Average sweep and span axis parameters of the soft dorsal and anal fin rays. (A) Timing of the first maximum sweep angle. (B) First maximum sweep angle. (C) Timing of the directional sweep transition. (D) Timing of the second maximum sweep angle. (E) Second maximum sweep angle. (F) Timing of the first maximum span axis angle. (G) First maximum span axis angle. $(\mathrm{H})$ Timing of the directional span axis transition. (I) Timing of the second maximum span axis angle. (J) Second maximum span axis angle. All timing parameters are in relation to the corresponding time event of the Mid-trunk

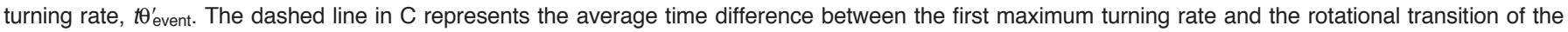
middle trunk, $\Delta t \theta_{\max 1}^{\prime}=t \theta_{\max 1}^{\prime}-t \theta_{\text {tr. }}^{\prime}$. For each parameter, Kendall's coefficient of concordance, $W$, and, if applicable, the multigroup coefficient of concordance, $\mathscr{W}$, are provided. Significant position effects are indicated in bold with an asterisk. Bars are means \pm 1 s.e.m., $N=9$ sequences from three fish.

and intra-fin forces transmitted via the fin webbing by the movement of adjacent fin rays. Over the course of a fast-start, the fin rays of the dorsal and anal fins demonstrated variations in their timing and magnitude for several kinematic parameters, based on position within the fin. Furthermore, these variations were generally in agreement with our expectations from previously discussed differences in musculoskeletal and biomechanical properties, supporting our hypothesis that there is functional regionalization within the dorsal and anal fins of the bluegill sunfish (Chadwell and Ashley-Ross, 2012). Although the data supported our hypothesis that fin rays are capable of resisting deflecting forces, the pattern in timing of the maximum sweep angles did not always match our original predictions about fin-ray resistance to lateral deflection relative to the axial body. This suggests that our original assumptions about the forces acting on the fin rays and/or the kinematic performance of the fin rays because of their resistance/susceptibility to lateral deflection are more complex than the simple model we had proposed.

\section{Spiny dorsal fin kinematics: sweep, span axis and elevation}

As expected, the spines generally resisted lateral deflection; however, the sweep parameters within $\mathrm{spD}$ were neither closely linked with the turning rate of Ant nor significantly correlated with spine position (Fig.7A-E, supplementary material TableS4), possibly because of the low degrees of freedom $($ d.f. $=2,3)$ and the conservative nature of the $\alpha$-adjustment. The timing of maximum sweep angle of the anterior three spines relative to maximum turning rate of Ant did not conform to our initial expectations of lagging behind the body due to hydrodynamic resistance. Rather than following the turning of Ant, as we had predicted, the timing of maximum sweep angles and directional transitions typically preceded that of Ant.

There are three potential explanations for this pattern. First, our initial hypothesis on the opposing hydrodynamic forces generated during the escape response may have been incorrect. During S1, rotation of the anterior trunk is delayed by ca. $10 \mathrm{~ms}$; however, this does not mean that there was no movement. As the head and posterior trunk segments rotate to the right, the anterior trunk consistently undergoes lateral translation to the left, i.e. a linear displacement rather than angular. Under this circumstance, opposing hydrodynamic force might passively deflect spines to the right, as was observed (Fig. 6A-D). Second, because of the restrictive nature of the spine joint, maximum lateral deflection (sweep) of the spines is limited and quickly met, with no further increase in deflection despite any increase in angular velocity and the resulting opposing forces exerted on the spines. And third, contractions of the inclinator muscles may allow spines to initiate lateral deflection independent of axial rotation and either actively resist, or generate hydrodynamic forces.

The timing of span axis rotation in $\mathrm{spD}$ was coupled with the timing of axial rotation of Ant, with no significant position effect or discernable pattern in the timing between spines (Fig. 7F,H,I). Maximum span axis rotation was correlated with position (Fig. 7G,J, supplementary material TableS4); however, because of the restrictive nature of the spine joints, it is unclear how much of the measured rotation may be due to the overall chordwise cupping of the fin membrane and lateral bend in the trunk (Fig. 3C,D). Future experiments might sever the fin membrane between spines to answer this question.

Elevation of the spines and increase in fin area always occurred within one or two frames after the onset of a C-start, reaching both maximum elevation and fin area well before the rotational transition of the anterior trunk (Fig. 8A-C). It is unclear to what extent the synchronized elevation is due to the activation of individual erector muscles associated with each spine (Chadwell and Ashley-Ross, 2012) versus elevation of the anteriormost spine (via its erector muscle) that passively moves the successive spines through the connection via the fin membrane. Timing of maximal elevation does 
not appear to be determined by rotation of the anterior trunk; rather, erection of the spines is most likely tied to the onset of the C-start, with maximum elevation and area typically reached within $10-15 \mathrm{~ms}$ from $T_{0}$ (data not shown). In the context of defense against a predator, rapid erection of sharp (and potentially gape-limiting) spines upon detection of a threat makes sense (Hoogland et al., 1956).

\section{Soft dorsal and anal fin kinematics: sweep, span axis and elevation}

As predicted, the magnitude and timing of all maximum sweep angles were significantly correlated with fin-ray position, with the posterior rays showing the greatest degree of deflection (Fig. 10B,E, supplementary material Tables S6, S7). Contrary to our simple hypothesis that the fin rays would follow the movement of their body segment, during S1, the anterior rays consistently achieved maximum sweep before Mid reached its maximum turning rate (Fig. 10A) and reversed direction earlier than Mid (Fig. 10C). During S2, maximum sweep of all rays preceded maximum turning rate of Mid (Fig. 10D). This supports the hypothesis that the fin rays (especially the anterior rays) are resistant to lateral deflection, and in fact are overcoming the opposing forces and are 'leaning into' the direction of movement. The hypotheses suggested for the spiny dorsal fin (previous section) may also apply to the anterior rays of sfD and sfA, which were found to have more restrictive joints than posterior rays (Chadwell and Ashley-Ross, 2012).

The consistency with which the timing of maximum sweep in Ry5 and Ry8 lagged behind the maximum turning rate of Mid (ca. $5 \mathrm{~ms}$; Fig. 10A) was perplexing. Sweep angle among these middle rays increased, even while axial turning rate was decreasing. Muscular activity may be responsible; a previous study found that muscle activity in the left dorsal inclinator muscles of bluegill sunfish, during right-handed $\mathrm{C}$-starts, began prior to the end of axial rotation of the trunk, whereas muscle activity of the right inclinators had ceased (Jayne et al., 1996). Among the rays tested, time of activation and duration of activity in the left inclinators were correlated with position, occurring sooner and lasting longer among the posterior rays tested. Our results match these findings, suggesting that as axial rotation to the right is slowing down, activity of the left inclinators is causing the fin rays to increase their sweep angle to the left, possibly stiffening them in preparation for opposing hydrodynamic forces during the subsequent rotation to the left.

Timing and maxima of the span axis rotations during S1 all showed significant position effects, though the relationship was not linear (Fig. 10F-H). With the exception of the maximum span axis rotation of the SfD fin rays, the more variable nature of S2 precluded finding significant position effects (Fig. 10I,J). Overall, the reversal in direction of span axis direction of the final ray (Ry12), like spD, indicates a cupping of the chordwise surface of the fin. The lack of a significant position effect in the maximal values of $\bar{\alpha}_{\max 1}$ for both sfD and sfA suggests that all the rays are capable of rotating about their span axis to the same degree.

Elevation in the soft fins followed a different pattern than that seen in the spiny dorsal fin (Fig. 8). As in the spiny dorsal fin, maximum elevation within sfD and sfA was significantly correlated with position (supplementary material Tables S5, S6), with maximum elevation decreasing posteriorly (Fig. 8E). Timing of maximum elevation and fin areas typically occurred during S2, after the rotational transition of the middle trunk; however, note that the timing of elevation of the first sfD element digitized (Ry2) closely follows the movement of the spine anterior to it $(\mathrm{sfD} \mathrm{Sp} 0=\mathrm{spD}$
DSp10), but more posterior rays show independence from $\mathrm{spD}$ movements (Fig. 8D). Timing was variable both within the fins as well as between fish, showing no position effect in either fin. As with the spines, the connective tissues of the fin membrane keeps the timing of maximum elevation and fin area closely uniform; however, the variability observed may indicate more independent control of elevation among the more flexible rays than seen in the spines.

Overall, the parameters associated with S2 typically showed the lowest degree of concordance between fish, particularly for the timing values. This is not surprising, as the later stage of escape responses shows the most variability in performance (Domenici and Blake, 1997; Law and Blake, 1996; Webb, 1978). Multigroup comparisons showed that, as predicted, kinematics were conserved between the soft dorsal and anal fins, with significant degrees of concordance between the two groups for all position effects found (supplementary material TableS7).

\section{Hydrodynamic role of the fins}

Traditionally, the role of the dorsal and anal spines has been seen as defensive, preventing capture by gape-limited predators and/or inflicting damage to predators that strike at that region (Hoogland et al., 1956). The immediate erection of the spines soon after the onset of an escape response, seen here, is consistent with that role. In the context of a study on the hydrodynamics of the escape response in bluegill (Tytell and Lauder, 2008), our results also support a role in the performance of the C-start by all three fins. In their study, three distinct jets of fluid, representing the momentum added to the water by the fish's movements, were produced by the body and tail: one at the trailing edge of the caudal fin, a second at the body in the location of the initial C-bend and a third at the mid-trunk, opposite from the second jet. They found that the second jet provided the primary source of thrust for accelerating the fish in its escape trajectory, with both the dorsal and anal fins contributing to the momentum of the second jet (ca. 37\% of total momentum, combined). A more recent study using computational fluid dynamics simulation, based on axial kinematics reported by Tytell and Lauder (Tytell and Lauder, 2008), confirmed the role of the three jets in accelerating the fish, although the separate contribution of mobile, flexible median fins was not addressed (Borazjani et al., 2012).

We suggest that the role of the spiny dorsal fin differs from that of the soft dorsal fin in the production of the fluid jet. As Tytell and Lauder showed, lateral bending of the body during S1 generates a suction force that draws fluid into the bend (Tytell and Lauder, 2008). The initial formation of the jet occurs in the area of the anterior trunk and the spiny dorsal fin. It is likely that the increase in lateral depth and synchronous chordwise bending of the erected $\mathrm{spD}$ adds to the volume of water entrained in the suction and raises the total momentum transmitted into the water. Although sfD and sfA may also contribute to the volume of water and the resulting momentum, the present results and curvature data presented in the companion paper (Chadwell et al., 2012) suggest that the traveling waves directed posteriorly along the fins (supplementary material Movie 1) function to accelerate the fluid during the end of S1, when peak acceleration often occurs, and may assist propulsion during S2.

We have demonstrated kinematic differences among the three median fins of bluegill during the $\mathrm{C}$-start escape response. The spiny dorsal fin appears to act to increase lateral depth and the amount of water that is acted upon during S1. The membranous connection 
between the stiff spiny and flexible soft regions of both dorsal and anal fins may allow for both enhanced mechanical support and effective water transfer posteriorly. The soft dorsal and anal fin rays undergo movements that suggest they shed jets of water that may increase thrust to move the fish away from the threat. Anterior and posterior regions of the soft fins show distinct kinematic patterns, indicating that these median appendages do not act as simple stiff plates, but rather as highly flexible and finely tuned control surfaces. Such detailed information will provide essential input to improve computational simulations of escape behavior, which are currently hampered by the lack of information regarding natural fin movements. Future studies that elucidate the active motor control over the fins and the role played by the connection between spiny and soft fins (by examining fish without continuous spiny and soft dorsal fins, e.g. largemouth bass, or interrupting the membranous connection between fins) will provide essential data to enable full understanding of the complex roles performed by the fins of rayfinned fishes.

\section{LIST OF SYMBOLS AND ABBREVIATIONS}

$A\langle x\rangle$

ARy\#

ASp\#

C

cT

$D$

DRy\#

$\mathrm{DSp} \#$

Fr frontal axis, a.k.a. normal to the frontal plane

L lateral axis, a.k.a. normal to the fin surface

Mid middle trunk

MSE mean square error

Op operculum

Post posterior trunk

$r \quad$ fin-ray identifier

Rs rostrum

S span axis of the fin surface

S1 stage 1 of the C-start

S2 stage 2 of the C-start

Seg body segment identifier

sfA soft region of the anal fin

sfD soft dorsal fin

Sg sagittal axis, a.k.a. normal to the sagittal plane

spD spiny dorsal fin

ssCOM stretched-straight center of mass

sT tangent to the spanwise curve

$t \quad$ time point during a C-start sequence

$T_{0} \quad$ time zero

TL total length

tr directional transition event, i.e. change in direction of rotation or orientation

$\underline{\mathrm{Tr}}$ transverse axis, a.k.a. normal to the transverse plane

$t \mathrm{X} \quad$ time of a given parameter, where $\mathrm{X}$ is the event of a given parameter

velocity of the ssCOM parallel to the fish trajectory

average span axis angle of a fin ray

$\Delta t \mathrm{X} \quad$ time difference between a given fin-ray parameter and its

corresponding axial event, where $\mathrm{X}$ is the event of a given parameter

$\bar{\phi} \quad$ average elevation of a fin ray

$\theta^{\prime} \quad$ turning rate, i.e. the first time derivative of yaw

$\bar{\omega} \quad$ average sweep angle of a fin ray

\section{ACKNOWLEDGEMENTS}

We thank Peter Madden for writing DigiMat, the custom video analysis program used in this study. We also thank two anonymous reviewers for constructive comments that improved the manuscript.

\section{FUNDING}

This work was supported by the National Science Foundation [grant IBN-0316331 to M.A.A.-R. and grant EFRI-0938043 to G.V.L.] and a Vecellio Fund Fellowship to B.A.C.

\section{REFERENCES}

Borazjani, I., Sotiropoulos, F., Tytell, E. D. and Lauder, G. V. (2012). Hydrodynamics of the bluegill sunfish $\mathrm{C}$-start escape response: three-dimensional simulations and comparison with experimental data. J. Exp. Biol. 215, 671-684

Canfield, J. G. and Rose, G. J. (1993). Activation of Mauthner neurons during prey capture. J. Comp. Physiol. A 172, 611-618.

Chadwell, B. A. and Ashley-Ross, M. A. (2012). Musculoskeletal morphology and regionalization within the dorsal and anal fins of bluegill sunfish (Lepomis macrochirus). J. Morphol. 273, 405-422.

Chadwell, B. A., Standen, E. M., Lauder, G. V. and Ashley-Ross, M. A. (2012) Median fin function during the escape response of bluegill sunfish (Lepomis macrochirus). II: Fin-ray curvature. J. Exp. Biol. 215, 2881-2890.

Domenici, P. and Blake, R. W. (1991). The kinematics and performance of the escape response in the angelfish (Pterophyllum eimekel). J. Exp. Biol. 156, 187-205.

Domenici, P. and Blake, R. W. (1997). The kinematics and performance of fish faststart swimming. J. Exp. Biol. 200, 1165-1178.

Eaton, R. C., Bombardieri, R. A. and Meyer, D. L. (1977). The Mauthner-initiated startle response in teleost fish. J. Exp. Biol. 66, 65-81.

Fernald, R. D. (1975). Fast body turns in a cichlid fish. Nature 258, 228-229.

Harper, D. G. and Blake, R. W. (1990). Fast-start performance of rainbow trout Salmo gairdneri and northern pike Esox lucius. J. Exp. Biol. 150, 321-342.

Hoogland, R., Morris, D. and Tinbergen, N. (1956). The spines of sticklebacks (Gasterosteus and Pygosteus) as means of defence against predators (Perca and Esox). Behaviour 10, 205-236.

Jayne, B. C. and Lauder, G. V. (1993). Red and white muscle activity and kinematics of the escape response of the bluegill sunfish during swimming. J. Comp. Physiol. A 173, 495-508.

Jayne, B. C., Lozada, G. F. and Lauder, G. V. (1996). Function of the dorsal fin in bluegill sunfish: motor patterns during four distinct locomotor behaviors. J. Morphol. 228, 307-326.

Kreyszig, E. (1991). Differential Geometry. New York: Dover Publications.

Lauder, G. V., Nauen, J. C. and Drucker, E. G. (2002). Experimental hydrodynamics and evolution: function of median fins in ray-finned fishes. Integr. Comp. Biol. 42, 1009-1017.

Lauder, G. V., Madden, P. G. A., Tangorra, J. L., Anderson, E. and Baker, T. V. (2011). Bioinspiration from fish for smart material design and function. Smart Mater. Struct. 20, 094014

Law, T. and Blake, R. (1996). Comparison of the fast-start performances of closely related, morphologically distinct threespine sticklebacks (Gasterosteus spp.). J. Exp. Biol. 199, 2595-2604.

Rand, D. M. and Lauder, G. V. (1981). Prey capture in the chain pickerel, Esox niger correlations between feeding and locomotor behavior. Can. J. Zool. 59, 1072-1078.

Rice, W. R. (1989). Analyzing tables of statistical tests. Evolution 43, 223-225.

Schriefer, J. E. and Hale, M. E. (2004). Strikes and startles of northern pike (Esox lucius): a comparison of muscle activity and kinematics between S-start behaviors. J. Exp. Biol. 207, 535-544.

Sokal, R. R. and Rohlf, F. J. (1981). Biometry. New York: W. H. Freeman

Spierts, I. L. and Leeuwen, J. L. (1999). Kinematics and muscle dynamics of C- and S-starts of carp (Cyprinus carpio L.). J. Exp. Biol. 202, 393-406.

Standen, E. M. and Lauder, G. V. (2005). Dorsal and anal fin function in bluegill sunfish Lepomis macrochirus: three-dimensional kinematics during propulsion and maneuvering. J. Exp. Biol. 208, 2753-2763.

Tytell, E. D. (2006). Median fin function in bluegill sunfish Lepomis macrochirus: streamwise vortex structure during steady swimming. J. Exp. Biol. 209, 1516-1534.

Tytell, E. D. and Lauder, G. V. (2008). Hydrodynamics of the escape response in bluegill sunfish, Lepomis macrochirus. J. Exp. Biol. 211, 3359-3369.

Vogel, S. (2003). Comparative Biomechanics: Life's Physical World. Princeton, NJ: Princeton University Press.

Wakeling, J. M. (2001). Biomechanics of fast-start swimming in fish. Comp. Biochem. Physiol. 131A, 31-40.

Walker, J. A. (1998). Estimating velocities and accelerations of animal locomotion: a simulation experiment comparing numerical differentiation algorithms. J. Exp. Biol. 201, 981-995.

Webb, P. W. (1976). The effect of size on the fast-start performance of rainbow trout Salmo cairdneri, and a consideration of piscivorous predator-prey interactions. $J$. Exp. Biol. 65, 157-177.

Webb, P. W. (1977). Effects of median-fin amputation on fast-start performance of rainbow trout (Salmo gairdneri). J. Exp. Biol. 68, 123-135.

Webb, P. W. (1978). Fast-start performance and body form in seven species of teleost fish. J. Exp. Biol. 74, 211-226.

Webb, P. W. and Skadsen, J. M. (1980). Strike tactics of Esox. Can. J. Zool. 58, $1462-1469$.

Weihs, D. (1973). The mechanism of rapid starting of slender fish. Biorheology 10, 343-350.

Zar, J. H. (1984). Biostatistical Analysis. Englewood Cliffs, NJ: Prentice Hall. 